MATHEMATICS OF COMPUTATION

Volume 79, Number 270, April 2010, Pages 1123-1144

S $0025-5718(09) 02287-\mathrm{X}$

Article electronically published on July 16, 2009

\title{
THE FIFTEEN THEOREM FOR UNIVERSAL HERMITIAN LATTICES OVER IMAGINARY QUADRATIC FIELDS
}

\author{
BYEONG MOON KIM, JI YOUNG KIM, AND POO-SUNG PARK
}

\begin{abstract}
We will introduce a method to get all universal Hermitian lattices over imaginary quadratic fields $\mathbb{Q}(\sqrt{-m})$ for all $m$. For each imaginary quadratic field $\mathbb{Q}(\sqrt{-m})$, we obtain a criterion on universality of Hermitian lattices: if a Hermitian lattice $L$ represents $1,2,3,5,6,7,10,13,14$ and 15, then $L$ is universal. We call this the fifteen theorem for universal Hermitian lattices. Note that the difference between Conway-Schneeberger's fifteen theorem and ours is the number 13. In addition, we determine the minimal rank of universal Hermitian lattices for all imaginary quadratic fields.
\end{abstract}

\section{INTRODUCTION}

The research on positive definite rational quadratic forms for which the represented integer set is as large as possible has its origins at the beginning of modern number theory. In 1770, Lagrange [19] found the famous four square theorem: the positive definite quadratic form $x_{1}^{2}+x_{2}^{2}+x_{3}^{2}+x_{4}^{2}$ represents all positive integers. Since then, his theorem has been generalized in many directions. One of the generalizations is to find all positive definite quadratic forms that represent all positive integers, which we call universal quadratic forms. The first breakthrough in this direction was made by Ramanujan 23. In 1917, he discovered all 55 quaternary diagonal universal forms, up to isometry. In 1927, Dickson [5] confirmed Ramanujan's list except for one form which was not universal and extended Ramanujan's results to nondiagonal forms. It was Dickson who called those forms universal. In 1948, Willerding 26 found 124 quaternary classical nondiagonal universal forms, up to isometry, and claimed that the list was complete. But her list was incomplete with some mistakes.

More generally, a positive definite quadratic form over a totally real number field is said to be universal if every totally positive integer of the field is represented by it. In 1928, Götzky [7] proved that $x_{1}^{2}+x_{2}^{2}+x_{3}^{2}+x_{4}^{2}$ is universal over $\mathbb{Q}(\sqrt{5})$. In 1941, Maass 20] proved the three square theorem, which states: the quadratic form $x_{1}^{2}+x_{2}^{2}+x_{3}^{2}$ is universal over $\mathbb{Q}(\sqrt{5})$. All positive definite ternary universal forms over real quadratic fields were determined in [3]. Further developments on universal

Received by the editor March 28, 2008 and, in revised form, April 14, 2009.

2000 Mathematics Subject Classification. Primary 11E39; Secondary 11E20, 11E41.

Key words and phrases. Universal Hermitian form.

The first named author was supported by the Korean Council for University Education, grant funded by Korean Government (MOEHRD) for 2006 Domestic Faculty Exchange.

The second and the third named authors were partially supported by $\mathrm{KRF}(2005-070-\mathrm{c} 00004)$.

(C)2009 American Mathematical Society Reverts to public domain 28 years from publication 
forms over totally real number fields were established by B. M. Kim (see [12, 13. and [14]).

If a positive definite Hermitian lattice over the ring of integers of an imaginary quadratic field represents all positive integers, we call it universal. In 1997, Earnest and Khosravani [6] found 13 universal binary Hermitian forms over imaginary quadratic fields of class number 1 . If the quadratic field over $\mathbb{Q}$ has a class number greater than 1, Iwabuchi 10 determined all universal binary Hermitian lattices over this field. After that, J.-H. Kim and P.-S. Park [17] added three binary Hermitian forms to the Earnest-Khosravani-Iwabuchi list and completed the list. Further generalizations of these results were made by P.-S. Park 22] and A. Rokicki 24 .

In 1993, Conway and Schneeberger announced the fifteen theorem for classical universal quadratic forms, which characterizes the universality by representability of a finite set of numbers, namely, 1, 2, 3, 5, 6, 7, 10, 14, and 15. Using the fifteen theorem, they corrected several mistakes in Willerding's list and announced the new and complete list of 204 quaternary classical universal forms up to isometry. They never published the original proof of this theorem, but we can find a sketch of their proof in 25]. B. M. Kim, M.-H. Kim and B.-K. Oh proved a 2-universality analogy in 15. Recently, Bhargava and Hanke enunciated that they proved the 290conjecture which characterizes the universality of (nonclassical) quadratic forms. That is, if a (nonclassical) quadratic form represents the 29 numbers, 1, 2, 3, 5, $6,7,10,13,14,15,17,19,21,22,23,26,29,30,31,34,35,37,42,58,93,110$, $145,203,290$, then it is universal (see 9]). One can use this theorem to prove the universality of a Hermitian lattice. But we succeeded in proving the universality of a Hermitian lattice without this big theorem. Moreover, our methods lead to a minimal set of critical numbers for each imaginary quadratic field.

In [4, Bhargava's generalization of the fifteen theorem was announced: for any infinite set $S$ of positive integers, there is a finite subset $S_{0}$ of $S$ such that any positive definite quadratic form that represents every element of $S_{0}$ represents all elements of $S$. Also, he found $S_{0}$ for some interesting sets $S$. In [16, B. M. Kim, M.-H. Kim and B.-K. Oh proved the finiteness theorem as a generalization of Bhargava's result: for any infinite set $S$ of positive definite quadratic forms of bounded rank, there is a finite subset $S_{0}$ of $S$ such that any positive definite quadratic form that represents every element of $S_{0}$ represents all of $S$. Nice survey papers related to these subjects are [18] and [8].

In this paper, first, we will suggest a matrix representation for nonfree Hermitian lattices. Due to this matrix representation, we can do escalation to find candidates of universal Hermitian lattices, including nonfree lattices over imaginary quadratic fields $\mathbb{Q}(\sqrt{-m})$. Second, we obtain a Conway-Schneeberger-Bhargava type criterion on universality of Hermitian lattices: if a Hermitian lattice $L$ represents $1,2,3$, $5,6,7,10,13,14$ and 15 , then $L$ is universal. Hence we call this theorem the fifteen theorem for universal Hermitian lattices. Following the language of the finiteness theorem for representability, $S$ is the set of all positive integers and $S_{0}=$ $\{1,2,3,5,6,7,10,13,14,15\}$. We will call the set $S_{0}$ a set of critical numbers. Even though the fifteen theorem and 290-theorem gives us the rough upper bound for the critical numbers that lie in between 15 to 290 , it is hard to figure out the set of critical numbers for each field. For each imaginary quadratic field $\mathbb{Q}(\sqrt{-m})$, we will give an optimal set of critical numbers by arithmetic calculation. For example, a Hermitian lattice $L$ over $\mathbb{Q}(\sqrt{-39})$ is universal if and only if $L$ represents 1 , 
$2,3,5,6,7,13$. In addition, we define the minimal rank $u_{m}$ as an invariant for each imaginary quadratic field $\mathbb{Q}(\sqrt{-m})$. It is the minimal rank of positive definite universal Hermitian lattices over $\mathbb{Q}(\sqrt{-m})$. In this paper, we determine $u_{m}$ completely for all imaginary quadratic fields $\mathbb{Q}(\sqrt{-m})$.

\section{Preliminaries}

The notation and terminology of O'Meara's book 21] will be adopted here. For the terminology specific to the Hermitian case, the paper [11] can be referred to. We begin by setting some additional notation that will remain in effect throughout this paper. Let $F$ denote the imaginary quadratic field $\mathbb{Q}(\sqrt{-m})$ for a positive squarefree integer $m$ with nontrivial $\mathbb{Q}$-involution and let $\mathcal{O}$ be the ring of integers of $F$. It is well known that $\mathcal{O}$ is generated by $\left\{1, \omega=\omega_{m}\right\}$ over $\mathbb{Z}$, where $\omega_{m}=\sqrt{-m}$ if $m \equiv 1,2(\bmod 4)$ or $\omega_{m}=\frac{1+\sqrt{-m}}{2}$ if $m \equiv 3(\bmod 4)$. By the term $\mathcal{O}$-lattice $L$ (or integral lattice $L$ over $F$ ), we will mean a finitely generated $\mathcal{O}$-module on the Hermitian space $(V, H)$ over $F$, where $V$ is an $n$-dimensional vector space over $F$ with the nondegenerate Hermitian form $H$. All lattices considered here will be assumed to be integral and positive definite in the sense that $H(x, y) \in \mathcal{O}$ for all $x, y \in L$ and $H(x):=H(x, x)>0$ for all $x \neq 0$. It follows from these assumptions that $H(x)$, called a (Hermitian) norm, is in $\mathbb{Z}$ for all $x \in L$.

As the ring of integers of an imaginary quadratic field is not generally a principal ideal domain, lattices do not need to be free. Let $\left\{v_{1}, \ldots, v_{n}\right\}$ be an $\mathcal{O}$-basis for $L$. In the case that $\mathcal{O}$ is a principal ideal domain, every Hermitian lattice is free. Therefore $L=\mathcal{O} v_{1}+\cdots+\mathcal{O} v_{n}$ and there is a function $f: \mathcal{O}^{n} \longrightarrow \mathbb{Z}$ defined by $f\left(x_{1}, \ldots, x_{n}\right)=$ $H\left(\sum x_{i} v_{i}\right)=\sum H\left(v_{i}, v_{j}\right) x_{i} \overline{x_{j}}$. Such a function will be referred to as a Hermitian form associated to $L$, and we can obtain an associated Hermitian matrix for $L$ by taking the $n \times n$ matrix whose entry is $H\left(v_{i}, v_{j}\right)$. If a basis $\left\{v_{1}, \ldots, v_{n}\right\}$ for $L$ is orthogonal, then the associated matrix of $L$ is denoted by $\left\langle H\left(v_{1}\right), \ldots, H\left(v_{n}\right)\right\rangle$. Similarly, in the case that $\mathcal{O}$ is not a principal ideal domain, there is a fractional ideal $\mathfrak{A}$ such that $L=\mathcal{O} v_{1}+\cdots+\mathcal{O} v_{n-1}+\mathfrak{A} v_{n}$ by [21, 81:5]. Since any ideal in $\mathcal{O}$ is generated by at most two elements, we can write $L=\mathcal{O} v_{1}+\cdots+\mathcal{O} v_{n-1}+(\alpha, \beta) \mathcal{O} v_{n}$ for some $\alpha, \beta \in \mathcal{O}$. Therefore, we have a Hermitian form $f: \mathcal{O}^{n-1} \times \mathcal{O} \longrightarrow \mathbb{Z}$ associated to $L$ such that $f\left(x_{1}, \ldots, x_{n}\right)=H\left(\sum_{i=1}^{n-1} x_{i} v_{i}+x_{n} \alpha v_{n}+x_{n} \beta v_{n}\right)$. Also, we have a Hermitian $(n+1) \times(n+1)$ matrix associated to $L$ as follows:

$$
\left(\begin{array}{cccc}
H\left(v_{1}, v_{1}\right) & \ldots & H\left(v_{1}, \alpha v_{n}\right) & H\left(v_{1}, \beta v_{n}\right) \\
\vdots & \ddots & \vdots & \vdots \\
H\left(\alpha v_{n}, v_{1}\right) & \ldots & H\left(\alpha v_{n}, \alpha v_{n}\right) & H\left(\alpha v_{n}, \beta v_{n}\right) \\
H\left(\beta v_{n}, v_{1}\right) & \ldots & H\left(\beta v_{n}, \alpha v_{n}\right) & H\left(\beta v_{n}, \beta v_{n}\right)
\end{array}\right)
$$

Note that this matrix is positive semi-definite, but this represents an $n$-ary positive definite Hermitian lattice.

Considering a $2 n$-dimensional vector space $\widetilde{V}$ over $\mathbb{Q}$ corresponding to $V$ as defined in [11, we can regard $(V, H)$ over $F$ as a $2 n$-dimensional quadratic space $\left(\widetilde{V}, B_{H}\right)$ such that $B_{H}(x, y)=\frac{1}{2}[H(x, y)+H(y, x)]=\frac{1}{2} \operatorname{Tr}_{F / \mathbb{Q}}(H(x, y))$. Analogously, by viewing $L$ as a $\mathbb{Z}$-lattice on $\left(\widetilde{V}, B_{H}\right)$ we can obtain a quadratic $\mathbb{Z}$-lattice $\widetilde{L}$ on $\widetilde{V}$ associated to a Hermitian $\mathcal{O}$-lattice $L$ on $V$, and also $\widetilde{f}\left(x_{1}, y_{1}, \ldots, x_{n}, y_{n}\right)=$ $f\left(x_{1}+\omega y_{1}, \ldots, x_{n}+\omega y_{n}\right)$ is an associated quadratic form in $2 n$-variables corresponding to this lattice $\widetilde{L}$. For convenience, we say that $\widetilde{f}$ is an associated quadratic form 
of $L$. For example, the associated quadratic form of the Hermitian lattice $\langle 1\rangle$ over $\mathbb{Q}(\sqrt{-m})$ is

$$
\left\{\begin{array}{lll}
x_{1}^{2}+m y_{1}^{2} & \text { if } m \equiv 1,2 & (\bmod 4), \\
x_{1}^{2}+x_{1} y_{1}+\frac{1+m}{4} y_{1}^{2} & \text { if } m \equiv 3 & (\bmod 4) .
\end{array}\right.
$$

To distinguish the matrix of a quadratic $\mathbb{Z}$-lattice $\widetilde{L}$ from the matrix of a Hermitian lattice $L$, we will add the subscript $\mathbb{Z}$ to the matrix of the quadratic $\mathbb{Z}$-lattice $\widetilde{L}$. Also, a Hermitian $\mathcal{O}$-lattice $L$ represents a $\mathbb{Z}$-lattice $M$ if and only if the associated quadratic form of $L$ represents $M$.

If $L$ is a universal lattice of rank $n$, then there are infinitely many universal lattices of rank $k(k>n)$ which contain $L$. Thus in order to obtain any meaningful finiteness result for such lattices, we should consider a new universal lattice. A universal lattice is called new when it does not contain any universal lattice of smaller rank.

For $m \equiv 1,2(\bmod 4)$, the associated quadratic lattice of a Hermitian lattice over $\mathbb{Q}(\sqrt{-m})$ is a classical $\mathbb{Z}$-lattice. Thus we can determine the universality of a Hermitian lattice over $\mathbb{Q}(\sqrt{-m})$ via applying the fifteen theorem. On the contrary, for $m \equiv 3(\bmod 4)$, the associated quadratic lattice of a Hermitian lattice over $\mathbb{Q}(\sqrt{-m})$ is a nonclassical $\mathbb{Z}$-lattice. Therefore, the recent big result of Bhargava and Hanke, the 290-conjecture, can be applied to prove the universality.

We adopt some notation from Conway-Sloane [4. The notation $p^{d}$ (resp. $p^{e}$ ) denotes an odd (resp. even) power of $p$; if $p=2, u_{k}$ denotes a unit of the form $8 n+k(k=1,3,5,7)$ and if $p$ is odd, $u_{+}$(resp. $\left.u_{-}\right)$denotes a unit which is a quadratic residue (resp. nonresidue) modulo $p$.

\section{MAin Results}

Theorem 1. For all positive square-free integers $m$, if a Hermitian lattice over $\mathbb{Q}(\sqrt{-m})$ represents the following critical numbers, then it is universal.

\begin{tabular}{l|l}
\hline critical numbers & $m$ \\
\hline 1,2 & 3,11 \\
1,3 & 1,7 \\
1,5 & 2 \\
$1,2,3$ & 5,19 \\
$1,2,3,5$ & 6 \\
$1,2,3,5,7$ & 15,23 \\
$1,2,3,5,6,7$ & 10,31 \\
$1,2,3,5,6,7,10$ & 13,14 \\
$1,2,3,5,6,7,13$ & 39 \\
$1,2,3,5,6,7,10,14$ & $35,43,51,59$ \\
$1,2,3,5,6,7,10,15$ & 55 \\
$1,2,3,5,6,7,10,14,15$ & otherwise \\
\hline
\end{tabular}

From this theorem, we have the criterion on the universality of Hermitian lattices.

Corollary 1 (The fifteen theorem for universal Hermitian lattices). If a positive definite Hermitian lattice represents 1, 2, 3, 5, 6, 7, 10, 13, 14, and 15, then it is universal.

Unlike the fifteen theorem for universal quadratic forms, this theorem involves the number 13 for the case of $\mathbb{Q}(\sqrt{-39})$. 
On the other hand, in the process of constructing universal Hermitian lattices over $\mathbb{Q}(\sqrt{-m})$, we can completely determine the minimal rank $u_{m}$ which is a kind of invariant for the imaginary quadratic field $\mathbb{Q}(\sqrt{-m})$. It is clear that $u_{m} \leq 4$ for all $m$.

Theorem 2. If we set the minimal rank

$$
u_{m}:=\min \{\operatorname{rank} L: L \text { is a universal Hermitian lattice over } \mathbb{Q}(\sqrt{-m})\}
$$

for each positive square-free integer $m$, then

\begin{tabular}{c|llllllllllll}
\hline$u_{m}$ & \multicolumn{10}{|c}{$m$} \\
\hline 2 & 1, & 2, & 3, & 5, & 6, & 7, & 10, & 11, & 15, & 19, & 23, & 31, \\
\hline 3 & 13, & 14, & 17, & 21, & 22, & 26, & 29, & 30, & 34, & 35, & 39, & 41, \\
& 43, & 46, & 47, & 51, & 55, & 59, & 71, & 79, & 83, & 87, & 91, & 95, \\
& 103, & 107, & 111, & 115, & 119, & 127, & 131, & 135, & 139, & 143, & 147, & 151, \\
& 155, & 159, & 167, & 171, & 175, & 179, & 183, & 187, & 191, & 199, & 207, & 215, \\
& 223, & & & & & & & & & & & \\
\hline 4 & 33, & 37, & 38, & 42, & 53, & 57, & 58, & 61, & 62, & 65, & 66, & 67, \\
& 69, & 70, & 73, & 74, & 77, & 78, & 82, & 85, & 86, & 89, & 93, & 94, \\
& 97, & 101, & 102, & 105, & 106, & 109, & 110, & 113, & 114, & 118, & 122, & 123, \\
& 129, & 130, & 133, & 134, & 137, & 138, & 141, & 142, & 145, & 146, & 149, & 154, \\
& 157, & 158, & 161, & 163, & 165, & 166, & 170, & 173, & 174, & 177, & 178, & 181, \\
& 182, & 185, & 186, & 190, & 193, & 194, & 195, & 197, & 201, & 202, & 203, & 205, \\
& 206, & 209, & 210, & 211, & 213, & 214, & 217, & 218, & 219, & 221, & 222, & 226, \\
& 227, & 229, & 230, & 231 & or & $m \geq$ & 235. & & & & & \\
\hline
\end{tabular}

Throughout the remainder of this paper, we will describe long proofs for these theorems. We will explain some notions preparatory to the proofs and then we will construct universal Hermitian lattices.

Note that $x \bar{x}+y \bar{y}+z \bar{z}+u \bar{u}$ represents all positive integers even when all variables take values in $\mathbb{Z}$. Thus it is obviously a quaternary universal Hermitian lattice over all imaginary quadratic fields. Since classical universal quadratic forms over $\mathbb{Z}$ were already classified, we do not need to investigate lattices of this kind. A Hermitian lattice is called inherited if its coefficients are all rational integers. If a Hermitian lattice is not inherited, we call it uninherited. We will mainly consider uninherited universal Hermitian lattices.

If a lattice $L$ is not universal, define the truant of $L$ to be the smallest positive integer not represented by $L$. An escalation of a nontrivial lattice $L$ is defined to be any lattice which is generated by $L$ and a vector whose norm is equal to the truant of $L$. Conway, Schneeberger and Bhargava suggested this escalation method for free lattices (see [25, [1]). In this article, we suggested the method for a matrix representation for nonfree Hermitian lattices (see section 2) and we use the escalation method to find candidates of universal Hermitian lattices including nonfree Hermitian lattices.

Let $L$ be a universal Hermitian lattice over $\mathbb{Q}(\sqrt{-m})$. Since $L$ represents 1 , $L \cong\langle 1\rangle \perp M$ for some lattice $M$. Since $2 \rightarrow L$, there are three incompatible cases: $2 \rightarrow\langle 1\rangle, 1 \rightarrow M$, or $2 \rightarrow M$. 
Case I. $2 \rightarrow\langle 1\rangle$ : In this case, $\alpha \bar{\alpha}=2$ has a solution for some $\alpha \in \mathcal{O}$. Let $\alpha=x_{1}+x_{2} \omega_{m}$. If $m \equiv 1,2(\bmod 4)$, then $\alpha \bar{\alpha}=x_{1}^{2}+m x_{2}^{2}=2$. So $m=1$ or 2. If $m \equiv 3(\bmod 4)$, then $\alpha \bar{\alpha}=x_{1}^{2}+x_{1} x_{2}+\frac{m+1}{4} x_{2}^{2}=2$. So $m=7$. Therefore all possible $m$ 's are 1,$2 ; 7$. We use a semicolon to distinguish $m \equiv 1,2(\bmod 4)$ and $m \equiv 3(\bmod 4)$. To represent from 1 through $5, L$ should have the following sublattices:

$\mathbb{Q}(\sqrt{-1}): \quad\langle 1,1\rangle,\langle 1,2\rangle,\langle 1,3\rangle$,

$\mathbb{Q}(\sqrt{-2}): \quad\langle 1,1\rangle,\langle 1,2\rangle,\langle 1,3\rangle,\langle 1,4\rangle,\langle 1,5\rangle$,

$\mathbb{Q}(\sqrt{-7}):\langle 1,1\rangle,\langle 1,2\rangle,\langle 1,3\rangle$.

These Hermitian lattices are all universal by [6] and [17, and we know that there are no other new Hermitian universal lattices over $\mathbb{Q}(\sqrt{-m})$ for $m=1,2 ; 7$. Hence we assume that $m \neq 1,2 ; 7$.

Case II. $2 \not \rightarrow\langle 1\rangle$ and $1 \rightarrow M$ : Then $L \cong\langle 1,1\rangle \perp K$ for some lattice $K$. Since 3 is a truant of the $\mathbb{Z}$-lattice $\langle 1,1\rangle_{\mathbb{Z}}$ and $L$ should represent 3 , we have four cases.

Case II-1. $3 \rightarrow\langle 1,1\rangle:$ In this case, $\alpha \bar{\alpha}+\beta \bar{\beta}=3$ has a solution for some $\alpha, \beta \in \mathcal{O}$, the possible $m$ 's are 3 or 11 . The universalities of $\langle 1,1\rangle$ over $\mathbb{Q}(\sqrt{-3})$ and $\mathbb{Q}(\sqrt{-11})$ were shown in $[6$. Assume that $m \neq 3,11$ for the following subcases.

Case II-2. $1 \rightarrow K$ : Then $L \cong\langle 1,1,1\rangle \perp N$ for some lattice $N$. Note that the truant of $\langle 1,1,1\rangle_{\mathbb{Z}}$ is 7 .

If $7 \rightarrow\langle 1,1,1\rangle$ over $\mathbb{Q}(\sqrt{-m})$, then we have $m=5,6 ; 15,19,23$. Since the sublattice $\langle 1,2\rangle$ of $\langle 1,1,1\rangle$ is universal over $\mathbb{Q}(\sqrt{-m})$ with $m=5 ; 19$ by [6] and [10], we do not need to consider the cases $m=5 ; 19$.

Case II-2(1). When $m=6$, the lattice $\langle 1,1,1\rangle$ represents a universal quadratic form $\langle 1,1,1,6\rangle_{\mathbb{Z}}$, i.e., the associated quadratic form $\langle 1,1,1,6,6,6\rangle_{\mathbb{Z}}$ of $\langle 1,1,1\rangle$ represents a universal quadratic form $\langle 1,1,1,6\rangle_{\mathbb{Z}}$. Thus the Hermitian lattice $\langle 1,1,1\rangle$ is also universal.

Case II-2(2). When $m=15,23,\langle 1,1,1\rangle$ can be written as an associated quadratic form over $\mathbb{Z}$ :

$$
x_{1}^{2}+x_{1} x_{2}+\frac{1+m}{4} x_{2}^{2}+y_{1}^{2}+y_{1} y_{2}+\frac{1+m}{4} y_{2}^{2}+z_{1}^{2}+z_{1} z_{2}+\frac{1+m}{4} z_{2}^{2} .
$$

This quadratic form has a sublattice $\langle 1,1,1\rangle_{\mathbb{Z}}$. It represents all positive integers except the form $2^{e} u_{7}$. If we set $x_{2}=2$ and $y_{2}=z_{2}=0$, then the quadratic form becomes

$$
\left(x_{1}+1\right)^{2}+y_{1}^{2}+z_{1}^{2}+m,
$$

and if we set $x_{2}=y_{2}=2$ and $z_{2}=0$, then the quadratic form becomes

$$
\left(x_{1}+1\right)^{2}+\left(y_{1}+1\right)^{2}+z_{1}^{2}+2 m .
$$

Let $n=2^{e} u_{7}$. If $n \geq 2 m$, then at least one of $n-m$ and $n-2 m$ is not of the form $2^{e} u_{7}$. Thus one of $n-m$ or $n-2 m$ can be represented by $\langle 1,1,1\rangle_{\mathbb{Z}}$. That is, $n \rightarrow\langle 1,1,1\rangle$ over $\mathbb{Q}(\sqrt{-m})$. It is easy to verify that $n \rightarrow\langle 1,1,1\rangle$ for $n<2 m$.

Hence we obtain new universal Hermitian lattices $\langle 1,1,1\rangle$ over $\mathbb{Q}(\sqrt{-m})$ with $m=6 ; 15,23$.

If $m \neq 5,6 ; 15,19,23,\langle 1,1,1\rangle$ cannot represent 7 over $\mathbb{Q}(\sqrt{-m})$ and thus $N$ should represent $1,2,3,4,5,6$ or 7 . But the quadratic form $\langle 1,1,1, a\rangle_{\mathbb{Z}}$ is universal for $a=1,2, \ldots, 7$ by [23]. That is, these lattices $\langle 1,1,1, a\rangle$ are inheritedly universal when $a=1,2, \ldots, 7$.

Case II-3. $1 \not \rightarrow K$ and $2 \rightarrow K$ : Then $L$ contains a ternary lattice $\langle 1,1,2\rangle$. Note that the truant of $\langle 1,1,2\rangle_{\mathbb{Z}}$ is 14 . 
Case II-3 a. $14 \rightarrow\langle 1,1,2\rangle:$ Then we have

$$
m=5,6,10,13,14 ; 15,19,23,31,35,39,43,47,51,55 .
$$

Since the sublattice $\langle 1,2\rangle$ of $\langle 1,1,2\rangle$ is universal over $\mathbb{Q}(\sqrt{-5})$ and $\mathbb{Q}(\sqrt{-19})$, $\langle 1,1,2\rangle$ is not a new universal Hermitian form over $\mathbb{Q}(\sqrt{-5})$ and $\mathbb{Q}(\sqrt{-19})$.

Case II-3 a(1). $m \not \equiv 3(\bmod 4)$ : Since $m=6,10,13,14$, the Hermitian lattice $\langle 1,1,2\rangle$ over $\mathbb{Q}(\sqrt{-m})$ represents a universal quadratic lattice $\langle 1,1,2, m\rangle_{\mathbb{Z}}$. Therefore $\langle 1,1,2\rangle$ is universal over $\mathbb{Q}(\sqrt{-m})$.

Case II-3 a(2). $m \equiv 3(\bmod 4): m=15,23,31,35,39,43,47,51,55$. For these cases we can write $\langle 1,1,2\rangle$ as an associated quadratic form over $\mathbb{Z}$ :

$$
x_{1}^{2}+x_{1} x_{2}+\frac{1+m}{4} x_{2}^{2}+y_{1}^{2}+y_{1} y_{2}+\frac{1+m}{4} y_{2}^{2}+2\left(z_{1}^{2}+z_{1} z_{2}+\frac{1+m}{4} z_{2}^{2}\right) .
$$

This form has a sublattice $\langle 1,1,2\rangle_{\mathbb{Z}}$ and it represents all positive integers except the form $2^{d} u_{7}$. If we set $x_{2}=2$ and $y_{2}=z_{2}=0$, then the quadratic form becomes

$$
\left(x_{1}+1\right)^{2}+y_{1}^{2}+2 z_{1}^{2}+m .
$$

Let $n=2^{d} u_{7}$. If $n \geq m$, then $n-m$ is not of the form $2^{d} u_{7}$ since $m$ is odd. Thus $n \rightarrow\langle 1,1,2\rangle$ over $\mathbb{Q}(\sqrt{-m})$, and we can show that $n \rightarrow\langle 1,1,2\rangle$ for $n<m$ by direct calculation.

Hence $\langle 1,1,2\rangle$ is a new universal Hermitian lattice over $\mathbb{Q}(\sqrt{-m})$ for

$$
m=6,10,13,14 ; 15,23,31,35,39,43,47,51,55 .
$$

Case II-3 b. $14 \not \rightarrow\langle 1,1,2\rangle$ : From $14 \not \rightarrow\langle 1,1,2\rangle$, we have

$$
\left\{\begin{array}{l}
m \geq 17 \text { if } m \neq \equiv 3 \quad(\bmod 4), \\
m \geq 59 \text { if } m \equiv 3 \quad(\bmod 4) .
\end{array}\right.
$$

Since $14 \rightarrow L, L$ should contain an escalation lattice $\ell$ of $\langle 1,1,2\rangle$ as follows:

$$
\ell \cong\left(\begin{array}{cccc}
1 & 0 & 0 & \alpha \\
0 & 1 & 0 & \beta \\
0 & 0 & 2 & \gamma \\
\bar{\alpha} & \bar{\beta} & \bar{\gamma} & 14
\end{array}\right) \cong\left(\begin{array}{cccc}
1 & 0 & 0 & 0 \\
0 & 1 & 0 & 0 \\
0 & 0 & 2 & \gamma \\
0 & 0 & \bar{\gamma} & 14-\alpha \bar{\alpha}-\beta \bar{\beta}
\end{array}\right)
$$

for some $\alpha, \beta, \gamma$. Note that $14-\alpha \bar{\alpha}-\beta \bar{\beta}=4,5,6,9,10,12,13,14$. The above lattice $\ell$ can be reduced once more to $\langle 1,1\rangle \perp\left(\begin{array}{cc}2 & a \\ \bar{a} & b\end{array}\right)$ with $a=0,1, \omega,-1+\omega$ and suitable $b$. If $a=0$ or 1 , then $\ell$ is one of the following:

$$
\begin{aligned}
& \langle 1,1\rangle \perp\left(\begin{array}{ll}
2 & 0 \\
0 & b
\end{array}\right) \text { with } b=2, \ldots, 14, \\
& \langle 1,1\rangle \perp\left(\begin{array}{ll}
2 & 1 \\
1 & b
\end{array}\right) \text { with } b=2,4,5,6,8,9,10,12,13,14 .
\end{aligned}
$$

These are all inherited universal lattices for all the above $m$ 's. Now consider the case of $a=\omega$ or $-1+\omega$. 
Case II-3 b(1). $m \not \equiv 3(\bmod 4)$ : From the positive semi-definiteness of $\ell$, we have $m=17,21,22,26$. In addition,

$$
\begin{aligned}
& \text { if } a=\omega, \text { then } b= \begin{cases}9,10,12,13,14, & \text { when } m=17, \\
12,13,14, & \text { when } m=21,22, \\
13,14, & \text { when } m=26,\end{cases} \\
& \text { and if } a=-1+\omega, \text { then } b= \begin{cases}9,10,12,13,14, & \text { when } m=17, \\
12,13,14, & \text { when } m=21,22, \\
14, & \text { when } m=26 .\end{cases}
\end{aligned}
$$

Since each $\ell$ represents a universal quaternary quadratic lattice $\langle 1,1,2, b\rangle_{\mathbb{Z}}$ or $\langle 1,1\rangle_{\mathbb{Z}} \perp\left(\begin{array}{ll}2 & 1 \\ 1 & b\end{array}\right)_{\mathbb{Z}}$ for each $b$, it is universal.

Case II-3 b(2). $m \equiv 3(\bmod 4)$ : From the positive semi-definiteness of $\ell$, we have $m=59,67,71,79,83,87,91,95,103,107,111$. If $\ell$ represents all positive integers smaller than $m$, then $\ell$ is universal, because $\ell$ contains $\langle 1,1,2\rangle$. We can easily check it by direct calculation.

Case II-4. $1,2 \nrightarrow \rightarrow K$ and $3 \rightarrow K$ : Then $L$ contains a ternary lattice $\langle 1,1,3\rangle$. Note that the truant of $\langle 1,1,3\rangle_{\mathbb{Z}}$ is 6 .

Case II-4 a. $6 \rightarrow\langle 1,1,3\rangle$ : Then we have

$$
m=5,6 ; 15,19,23 .
$$

Case II-4 a(1). $m \not \equiv 3(\bmod 4)$ : Since $m=5$ or 6 , the Hermitian lattice $\langle 1,1,3\rangle$ represents a universal quadratic form $\langle 1,1,3,5\rangle_{\mathbb{Z}}$ or $\langle 1,1,3,6\rangle_{\mathbb{Z}}$. Thus $\langle 1,1,3\rangle$ is universal over $\mathbb{Q}(\sqrt{-m})$.

Case II-4 a(2) $\cdot m \equiv 3(\bmod 4)$ : We have that $\langle 1,1,3\rangle_{\mathbb{Z}}$ can represent all positive integers except the form $3^{d} u_{-}$. Let $n=3^{d} u_{-}$.

First consider the case of $m=15$. The associated quadratic form of $\langle 1,1,3\rangle$ is a nonclassical quadratic form

$$
x_{1}^{2}+x_{1} x_{2}+4 x_{2}^{2}+y_{1}^{2}+y_{1} y_{2}+4 y_{2}^{2}+3 z_{1}^{2}+3 z_{1} z_{2}+12 z_{2}^{2} .
$$

Note that if $x_{2}=2$ and $y_{2}=z_{2}=0$, then the form becomes

$$
\left(x_{1}+1\right)^{2}+y_{1}^{2}+3 z_{1}^{2}+15,
$$

and if $x_{2}=y_{2}=2$ and $z_{2}=0$, then the form becomes

$$
\left(x_{1}+1\right)^{2}+\left(y_{1}+1\right)^{2}+3 z_{1}^{2}+30 .
$$

If $n \geq 30$, then at least one of $n-15$ and $n-30$ is not of the form $3^{d} u_{-}$. That is, $n$ can be represented by $\langle 1,1,3\rangle$. It is easily verified that $\langle 1,1,3\rangle$ represents all positive integers less than 30 . Thus it is a new universal Hermitian lattice over $\mathbb{Q}(\sqrt{-15})$.

Similarly, it can be shown that $\langle 1,1,3\rangle$ is a new universal Hermitian lattice over $\mathbb{Q}(\sqrt{-m})$ with $m=19,23$ by checking $n-19$ and $n-23$.

Case II-4 b. $6 \not \rightarrow\langle 1,1,3\rangle$ : From $6 \not \rightarrow\langle 1,1,3\rangle$, we have

$$
\left\{\begin{array}{l}
m \geq 10 \text { if } m \neq \equiv \quad(\bmod 4), \\
m \geq 31 \text { if } m \equiv 3 \quad(\bmod 4) .
\end{array}\right.
$$


Since $6 \rightarrow L, L$ should contain an escalation lattice $\ell$ of $\langle 1,1,3\rangle$ as follows:

$$
\ell \cong\left(\begin{array}{cccc}
1 & 0 & 0 & \alpha \\
0 & 1 & 0 & \beta \\
0 & 0 & 3 & \gamma \\
\bar{\alpha} & \bar{\beta} & \bar{\gamma} & 6
\end{array}\right) \cong\left(\begin{array}{cccc}
1 & 0 & 0 & 0 \\
0 & 1 & 0 & 0 \\
0 & 0 & 3 & \gamma \\
0 & 0 & \bar{\gamma} & 6-\alpha \bar{\alpha}-\beta \bar{\beta}
\end{array}\right)
$$

for some $\alpha, \beta, \gamma$. Note that $6-\alpha \bar{\alpha}-\beta \bar{\beta}=4,5,6$. Hence the above lattice $\ell$ can be reduced to $\langle 1,1\rangle \perp\left(\begin{array}{cc}3 & a \\ \bar{a} & b\end{array}\right)$ with $a=0,1, \omega, 1+\omega,-1+\omega$ and suitable $b$. If $a=0$ or 1 , then $b=3,4,5,6$ and $\ell$ is inherited and universal over $\mathbb{Q}(\sqrt{-m})$ for all the above $m$. Now assume that $a=\omega, 1+\omega$ or $-1+\omega$.

Case II-4 b(1) $m \not \equiv 3(\bmod 4)$ : From the positive semi-definiteness of $\ell$, we have

$$
m=10,13,14,17 .
$$

In addition, if $a=\omega, 1+\omega$ or $-1+\omega$, then

$$
b= \begin{cases}4,5,6, & \text { when } m=10, \\ 5,6, & \text { when } m=13,14, \\ 6, & \text { when } m=17 .\end{cases}
$$

Since each $\ell$ represents a universal quaternary quadratic lattice $\langle 1,1,3, b\rangle_{\mathbb{Z}}$ or $\langle 1,1\rangle_{\mathbb{Z}} \perp\left(\begin{array}{ll}3 & 1 \\ 1 & b\end{array}\right)_{\mathbb{Z}}$ for each $b$, it is a universal Hermitian lattice.

Case II-4 $\mathbf{b}(\mathbf{2}) \cdot m \equiv 3(\bmod 4)$ : From the positive semi-definiteness of $\ell$, we have $m=31,35,39,43,47,51,55,59,67,71$. We will apply the same argument in Case II- 4 a(2) for these $m$ 's. If $m=39,51$, then it is enough to check that each $\ell$ represents all positive integers smaller than $2 m$. If $m=31,35,43,47,55,59,67,71$, then it is enough to check whether $\ell$ represents all positive integers smaller than $m$. We can easily check this by direct calculation; hence $\ell$ is a universal Hermitian lattice.

Case III. $2 \nrightarrow \rightarrow\langle 1\rangle, 1 \nrightarrow \rightarrow M$ and $2 \rightarrow M$ : Then $L$ contains a binary lattice $\langle 1,2\rangle$. Note that the truant of $\langle 1,2\rangle_{\mathbb{Z}}$ is 5 . When $m=5 ; 3,11,19,\langle 1,2\rangle$ represents 5 and $\langle 1,2\rangle$ is universal for these $m$ 's by [6] and [10]. Thus through the Case III, we may assume $m \neq 5 ; 3,11,19$. Since $5 \rightarrow L$, the escalation lattice of $\langle 1,2\rangle$ is

$$
\left(\begin{array}{ccc}
1 & 0 & \alpha \\
0 & 2 & \beta \\
\bar{\alpha} & \bar{\beta} & 5
\end{array}\right) \cong\left(\begin{array}{ccc}
1 & 0 & 0 \\
0 & 2 & \beta \\
0 & \bar{\beta} & 5-\alpha \bar{\alpha}
\end{array}\right)
$$

for some $\alpha, \beta$. This lattice can be reduced to $\langle 1\rangle \perp\left(\begin{array}{cc}2 & a \\ \bar{a} & b\end{array}\right)$ with $a=0,1, \omega$ or $-1+\omega$ and suitable $b$. If $a=0,1$, then the escalation lattices are

$$
\langle 1,2,2\rangle,\langle 1,2,3\rangle,\langle 1,2,4\rangle,\langle 1,2,5\rangle,\langle 1\rangle \perp\left(\begin{array}{ll}
2 & 1 \\
1 & 4
\end{array}\right) \text { and }\langle 1\rangle \perp\left(\begin{array}{ll}
2 & 1 \\
1 & 5
\end{array}\right)
$$

for all the above $m$ 's. Also, if $a=\omega$ or $-1+\omega$, then the escalation lattice $\langle 1\rangle \perp$ $\left(\begin{array}{ll}2 & a \\ \bar{a} & b\end{array}\right)$ over $\mathbb{Q}(\sqrt{-m})$ satisfies the conditions of Table 1, up to isometry.

Now, we will treat each lattice in Case III-1 to Case III-7. 
TABLE 1. Conditions for $\langle 1\rangle \perp\left(\begin{array}{cc}2 & a \\ \bar{a} & b\end{array}\right)$

\begin{tabular}{l|l|l}
\hline $\mathbb{Q}(\sqrt{-m})$ & $a$ & $b$ \\
\hline $\mathbb{Q}(\sqrt{-6})$ & $\omega_{6}$ & $3,4,5$, \\
& $-1+\omega_{6}$ & 4,5, \\
$\mathbb{Q}(\sqrt{-10})$ & $\omega_{10}$ & 5, \\
$\mathbb{Q}(\sqrt{-15})$ & $\omega_{15}$ & $2,3,4,5$, \\
& $-1+\omega_{15}$ & $3,4,5$, \\
$\mathbb{Q}(\sqrt{-23})$ & $\omega_{23}$ & $3,4,5$, \\
& $-1+\omega_{23}$ & $3,4,5$, \\
$\mathbb{Q}(\sqrt{-31})$ & $\omega_{31}$ & 4,5, \\
& $-1+\omega_{31}$ & 4,5, \\
$\mathbb{Q}(\sqrt{-35})$ & $\omega_{35}$ & 5, \\
$\mathbb{Q}(\sqrt{-39})$ & $-1+\omega_{35}$ & 5, \\
& $\omega_{39}$ & 5, \\
& $-1+\omega_{39}$ & 5. \\
\hline
\end{tabular}

Case III-1. $\langle 1,2,2\rangle \rightarrow L$ : Note that $\langle 1,2,2\rangle$ represents a quadratic lattice $\langle 1,2,2\rangle_{\mathbb{Z}}$ whose truant is 7 .

Case III-1 a. $7 \rightarrow\langle 1,2,2\rangle$ : Then we have $m=6$ and $\langle 1,2,2\rangle$ is a new universal Hermitian lattice as $\langle 1,2,2\rangle$ represents a universal quadratic lattice $\langle 1,2,2,6\rangle_{\mathbb{Z}}$. Through the Case III-1, we may assume $m \neq 6$.

Case III-1 b. $7 \not \rightarrow\langle 1,2,2\rangle$ : Then $L$ should contain an escalation lattice $\ell$ of $\langle 1,2,2\rangle$ as follows:

$$
\ell \cong\left(\begin{array}{cccc}
1 & 0 & 0 & \alpha \\
0 & 2 & 0 & \beta \\
0 & 0 & 2 & \gamma \\
\bar{\alpha} & \bar{\beta} & \bar{\gamma} & 7
\end{array}\right)
$$

for some $\alpha, \beta, \gamma$.

Case III-1 b(1). $m \not \equiv 3(\bmod 4)$ : Then $\ell$ represents a quadratic lattice

$$
\ell^{\prime}=\left(\begin{array}{cccc}
1 & 0 & 0 & \operatorname{Re} \alpha \\
0 & 2 & 0 & \operatorname{Re} \beta \\
0 & 0 & 2 & \operatorname{Re} \gamma \\
\operatorname{Re} \alpha & \operatorname{Re} \beta & \operatorname{Re} \gamma & 7
\end{array}\right)_{\mathbb{Z}} .
$$

There are 16 lattices of the above form up to isometry and they are all universal by the fifteen theorem.

Case III-1 b(2) $\cdot m \equiv 3(\bmod 4)$ : From the positive semi-definiteness, the $\ell$ 's are all inherited for $m \geq 59$. Then, since $\ell$ represents the above quadratic lattice $\ell^{\prime}$, $\ell$ is universal. We may assume that $m=15,23,31,35,39,43,47,51,55$. Note that $\langle 1,2,2\rangle_{\mathbb{Z}}$ represents all positive integers only except the form $2^{e} u_{7}$ and $\langle 1,2,2\rangle$ represents $\langle 1,2,2, m\rangle_{\mathbb{Z}}$.

Let $n=2^{e} u_{7}$. If $n \geq 4 m$, then at least one of $n-m$ and $n-4 m$ is not of the form $2^{e} u_{7}$. Thus $n \rightarrow\langle 1,2,2\rangle_{\mathbb{Z}}$. If $n<4 m$, then we can show that $n$ is represented by each $\ell$ by direct calculation. Hence the universality of $\ell$ is proved.

Case III-2. $\langle 1,2,3\rangle \rightarrow L$ : Note that $\langle 1,2,3\rangle$ represents a quadratic lattice $\langle 1,2,3\rangle_{\mathbb{Z}}$ whose truant is 10 . 
Case III-2 a. $10 \rightarrow\langle 1,2,3\rangle:$ Then we have $m=6,10 ; 15,23,31,39$.

Case III-2 a(1). $m \not \equiv 3(\bmod 4)$ : Since $m=6$ or 10 , the Hermitian lattice $\langle 1,2,3\rangle$ represents a universal quadratic lattice $\langle 1,2,3,6\rangle_{\mathbb{Z}}$ or $\langle 1,2,3,10\rangle_{\mathbb{Z}}$. Thus the universality of the Hermitian lattice $\langle 1,2,3\rangle$ over $\mathbb{Q}(\sqrt{-m})$ is proved.

Case III-2 a(2). $m \equiv 3(\bmod 4)$ : Then the associated quadratic form of $\langle 1,2,3\rangle$ is

$$
x_{1}^{2}+x_{1} x_{2}+\frac{1+m}{4} x_{2}^{2}+2\left(y_{1}^{2}+y_{1} y_{2}+\frac{1+m}{4} y_{2}^{2}\right)+3\left(z_{1}^{2}+z_{1} z_{2}+\frac{1+m}{4} z_{2}^{2}\right) .
$$

If we set $x_{2}=2$ and $y_{2}=z_{2}=0$, then the quadratic form becomes $\langle 1,2,3\rangle_{\mathbb{Z}}+m$. Note that the quadratic lattice $\langle 1,2,3\rangle_{\mathbb{Z}}$ represents all positive integers except the form $2^{d} u_{5}$. If $n=2^{d} u_{5} \geq m$, then $n-m$ is not of the form $2^{d} u_{5}$ and hence $n-m \rightarrow\langle 1,2,3\rangle_{\mathbb{Z}}$. It is easily verified that $n \rightarrow\langle 1,2,3\rangle$ for $n<m$ by direct calculation. Hence the Hermitian lattice $\langle 1,2,3\rangle$ is universal. From now through the Case III-2, we may assume that $m \neq 6,10 ; 15,23,31,39$.

Case III-2 b. $10 \nrightarrow\langle\langle 1,2,3\rangle$ : The next escalation lattice $\ell$ of $\langle 1,2,3\rangle$ is of the form

$$
\ell \cong\left(\begin{array}{cccc}
1 & 0 & 0 & \alpha \\
0 & 2 & 0 & \beta \\
0 & 0 & 3 & \gamma \\
\bar{\alpha} & \bar{\beta} & \bar{\gamma} & 10
\end{array}\right)
$$

for some $\alpha, \beta, \gamma$.

Case III-2 b(1). $m \not \equiv 3(\bmod 4)$ : Then the lattice $\ell$ represents a quadratic lattice

$$
\ell^{\prime}=\left(\begin{array}{cccc}
1 & 0 & 0 & \operatorname{Re} \alpha \\
0 & 2 & 0 & \operatorname{Re} \beta \\
0 & 0 & 3 & \operatorname{Re} \gamma \\
\operatorname{Re} \alpha & \operatorname{Re} \beta & \operatorname{Re} \gamma & 10
\end{array}\right)_{\mathbb{Z}} .
$$

There are 28 lattices of this type up to isometry, and we know that they are all universal by the fifteen theorem.

Case III-2 b(2). $m \equiv 3(\bmod 4)$ : From the condition of positive semi-definiteness, the escalation lattices $\ell$ of $\langle 1,2,3\rangle$ are all inherited for $m \geq 123$. Then since $\ell$ represents the above quadratic lattice $\ell^{\prime}, \ell$ is universal. We may assume that $m=35,43,47, \ldots, 119$. Note that $\langle 1,2,3\rangle_{\mathbb{Z}}$ represents all positive integers except only the form $2^{d} u_{5}$. If $n=2^{d} u_{5} \geq m$, then $n-m$ is not of the form $2^{d} u_{5}$. Thus $n \rightarrow\langle 1,2,3\rangle_{\mathbb{Z}}$. If $n<m$, then we can show that $n$ is represented by each $\ell$ by direct calculation. Hence the universality of $\ell$ over $\mathbb{Q}(\sqrt{-m})$ is proved.

Case III-3. $\langle 1,2,4\rangle \rightarrow L$ : Note that $\langle 1,2,4\rangle$ represents a quadratic lattice $\langle 1,2,4\rangle_{\mathbb{Z}}$ whose truant is 14 .

Case III-3 a. $14 \rightarrow\langle 1,2,4\rangle:$ Then we have $m=6,10,13,14 ; 15,23,31,39,47$, 55 .

Case III-3 a(1). $m \not \equiv 3(\bmod 4)$ : Since $m=6,10,13,14,\langle 1,2,4\rangle$ over $\mathbb{Q}(\sqrt{-m})$ represents a universal quadratic form $\langle 1,2,4, m\rangle_{\mathbb{Z}}$. Hence the universality of $\langle 1,2,4\rangle$ over $\mathbb{Q}(\sqrt{-m})$ is proved.

Case III-3 a (2) $m \equiv 3(\bmod 4)$ : The associated quadratic form of $\langle 1,2,4\rangle$ is

$$
x_{1}^{2}+x_{1} x_{2}+\frac{1+m}{4} x_{2}^{2}+2\left(y_{1}^{2}+y_{1} y_{2}+\frac{1+m}{4} y_{2}^{2}\right)+4\left(z_{1}^{2}+z_{1} z_{2}+\frac{1+m}{4} z_{2}^{2}\right) .
$$


If we set $x_{2}=2$ and $y_{2}=z_{2}=0$, then the quadratic form becomes $\langle 1,2,4\rangle_{\mathbb{Z}}+m$. Note that the quadratic lattice $\langle 1,2,4\rangle_{\mathbb{Z}}$ represents all positive integers except the form $2^{d} u_{7}$. If $n=2^{d} u_{7} \geq m$, then $n-m$ is represented by $\langle 1,2,4\rangle_{\mathbb{Z}}$ and hence $n \rightarrow\langle 1,2,4\rangle$. It is easily verified that $\langle 1,2,4\rangle$ represents $n$ for $n<m$. Hence $\langle 1,2,4\rangle$ is universal over $\mathbb{Q}(\sqrt{-m})$. From now through the Case III-3 b, we may assume that $m \neq 6,10,13,14 ; 15,23,31,39,47,55$.

Case III-3 b. $14 \not \rightarrow\langle 1,2,4\rangle$ : The next escalation lattice $\ell$ of $\langle 1,2,4\rangle$ is of the form

$$
\ell \cong\left(\begin{array}{cccc}
1 & 0 & 0 & \alpha \\
0 & 2 & 0 & \beta \\
0 & 0 & 4 & \gamma \\
\bar{\alpha} & \bar{\beta} & \bar{\gamma} & 14
\end{array}\right)
$$

for some $\alpha, \beta, \gamma$.

Case III-3 b(1). $m \not \equiv 3(\bmod 4)$ : Then the lattice $\ell$ represents a quadratic lattice

$$
\ell^{\prime}=\left(\begin{array}{cccc}
1 & 0 & 0 & \operatorname{Re} \alpha \\
0 & 2 & 0 & \operatorname{Re} \beta \\
0 & 0 & 4 & \operatorname{Re} \gamma \\
\operatorname{Re} \alpha & \operatorname{Re} \beta & \operatorname{Re} \gamma & 14
\end{array}\right)_{\mathbb{Z}}
$$

There are 54 lattices of this type up to isometry and they are all universal by the fifteen theorem.

Case III-3 b(2). $m \equiv 3(\bmod 4)$ : From the positive semi-definiteness, the escalation lattices are all inherited for $m \geq 227$. Since $\ell$ represents the above quadratic lattice $\ell^{\prime}, \ell$ is universal. Thus we may assume that $m=35,43,51,59,67$, $71,79,83,87, \ldots, 223$. Note that $\langle 1,2,4\rangle_{\mathbb{Z}}$ represents all positive integers except only the form $2^{d} u_{7}$. We have that $n-m \rightarrow\langle 1,2,4\rangle_{\mathbb{Z}}$ implies $n \rightarrow\langle 1,2,4\rangle$. If $n=2^{d} u_{7} \geq m$, then $n-m$ is not of the form $2^{d} u_{7}$. Thus $n \rightarrow\langle 1,2,4\rangle_{\mathbb{Z}}$. By direct calculation it can be verified that $n \rightarrow \ell$ for $n<m$. Hence the universality of $\ell$ over $\mathbb{Q}(\sqrt{-m})$ is proved.

Case III-4. $\langle 1,2,5\rangle \rightarrow L$ : Note that $\langle 1,2,5\rangle$ represents a quadratic lattice $\langle 1,2,5\rangle_{\mathbb{Z}}$ whose truant is 10 .

Case III-4 a. $10 \rightarrow\langle 1,2,5\rangle$ : Then we should have $m=6,10 ; 15,23,31,39$.

Case III-4 a(1). $m \not \equiv 3(\bmod 4)$ : Since $m=6,10,\langle 1,2,5\rangle$ over $\mathbb{Q}(\sqrt{-m})$ represents a quaternary universal quadratic lattice $\langle 1,2,5, m\rangle_{\mathbb{Z}}$. Hence $\ell$ is universal.

Case III-4 a(2). $m \equiv 3(\bmod 4)$ : The associated quadratic form of $\langle 1,2,5\rangle$ is

$$
x_{1}^{2}+x_{1} x_{2}+\frac{1+m}{4} x_{2}^{2}+2\left(y_{1}^{2}+y_{1} y_{2}+\frac{1+m}{4} y_{2}^{2}\right)+5\left(z_{1}^{2}+z_{1} z_{2}+\frac{1+m}{4} z_{2}^{2}\right) .
$$

If we set $x_{2}=2$ and $y_{2}=z_{2}=0$, then the quadratic form becomes $\langle 1,2,5\rangle_{\mathbb{Z}}+m$. Similarly $\langle 1,2,5\rangle_{\mathbb{Z}}+2 m$ and $\langle 1,2,5\rangle_{\mathbb{Z}}+3 m$ are also obtained. The quadratic lattice $\langle 1,2,5\rangle_{\mathbb{Z}}$ represents all positive integers except the form $5^{d} u_{-}$. If $m=15$ and $n \geq 3 m$, then $n-m, n-2 m$, or $n-3 m$ is represented by $\langle 1,2,5\rangle_{\mathbb{Z}}$. If $m=23,31,39$ or $n=5^{d} u_{-} \geq 2 m$, then $n-m$ or $n-2 m$ is represented by $\langle 1,2,5\rangle_{\mathbb{Z}}$. It is easily verified that $\langle 1,2,5\rangle$ represents $n$ for $n<3 m$ or $n<2 m$. Hence $\langle 1,2,5\rangle$ is universal. From now through the Case III- $4 \mathrm{~b}$, we may assume that $m \neq 6,10 ; 15,23,31,39$. 
Case III-4 b. $10 \not \rightarrow\langle 1,2,5\rangle$ : The next escalation lattice $\ell$ of $\langle 1,2,5\rangle$ is of the form

$$
\ell \cong\left(\begin{array}{cccc}
1 & 0 & 0 & \alpha \\
0 & 2 & 0 & \beta \\
0 & 0 & 5 & \gamma \\
\bar{\alpha} & \bar{\beta} & \bar{\gamma} & 10
\end{array}\right)
$$

for some $\alpha, \beta, \gamma$.

Case III-4 b(1). $m \not \equiv 3(\bmod 4)$ : From the positive semi-definiteness, $\ell$ is inherited if $m \geq 53$. The lattice $\ell$ represents a quadratic lattice

$$
\left(\begin{array}{cccc}
1 & 0 & 0 & \operatorname{Re} \alpha \\
0 & 2 & 0 & \operatorname{Re} \beta \\
0 & 0 & 5 & \operatorname{Re} \gamma \\
\operatorname{Re} \alpha & \operatorname{Re} \beta & \operatorname{Re} \gamma & 10
\end{array}\right)_{\mathbb{Z}}
$$

There are 32 quadratic lattices of this type up to isometry. Among these quadratic lattices, 28 quadratic lattices are universal by the fifteen theorem, but the following 4 quadratic lattices are not. The truants of the following 4 quaternary quadratic lattices are all 15 :

$$
\langle 1\rangle_{\mathbb{Z}} \perp\left(\begin{array}{lll}
2 & 0 & 0 \\
0 & 5 & 0 \\
0 & 0 & 5
\end{array}\right)_{\mathbb{Z}},\langle 1\rangle_{\mathbb{Z}} \perp\left(\begin{array}{lll}
2 & 0 & 1 \\
0 & 5 & 1 \\
1 & 1 & 5
\end{array}\right)_{\mathbb{Z}},\langle 1\rangle_{\mathbb{Z}} \perp\left(\begin{array}{lll}
2 & 0 & 1 \\
0 & 5 & 2 \\
1 & 2 & 8
\end{array}\right)_{\mathbb{Z}},\langle 1\rangle_{\mathbb{Z}} \perp\left(\begin{array}{lll}
2 & 0 & 1 \\
0 & 5 & 1 \\
1 & 1 & 9
\end{array}\right)_{\mathbb{Z}} .
$$

Now assume that $\ell$ represents one of the above quadratic lattices. If $m=13$ or 14 , the Hermitian lattice $\ell$ represents 15 and hence they are universal. If $m \neq 13,14$ and $\ell$ is uninherited, then $\ell$ is one of the following:

$$
\begin{aligned}
& \langle 1\rangle \perp\left(\begin{array}{ccc}
2 & 0 & 0 \\
0 & 5 & \omega_{m} \\
0 & \bar{\omega}_{m} & 5
\end{array}\right) \quad \text { if } m=17,21,22, \\
& \langle 1\rangle \perp\left(\begin{array}{ccc}
2 & 0 & 1 \\
0 & 5 & 1 \pm \omega_{m} \\
1 & 1 \pm \bar{\omega}_{m} & 5
\end{array}\right) \quad \text { if } m=17,21 \\
& \langle 1\rangle \perp\left(\begin{array}{ccc}
2 & 0 & 1 \\
0 & 5 & 2 \pm \omega_{m} \\
1 & 2 \pm \bar{\omega}_{m} & 8
\end{array}\right) \quad \text { if } m=17,21,22,26,29,30,33 \\
& \langle 1\rangle \perp\left(\begin{array}{ccc}
2 & 0 & 1 \\
0 & 5 & 1 \pm \omega_{m} \\
1 & 1 \pm \bar{\omega}_{m} & 9
\end{array}\right) \quad \text { if } m=17,21,22,26,29,30,33,34,37,38,41
\end{aligned}
$$

Among the above lattices, only the following lattices represent 15 . Hence they are universal by the fifteen theorem.

$$
\begin{array}{rcc}
\langle 1\rangle \perp\left(\begin{array}{ccc}
2 & 0 & 0 \\
0 & 5 & \omega_{22} \\
0 & \bar{\omega}_{22} & 5
\end{array}\right), & \langle 1\rangle \perp\left(\begin{array}{ccc}
2 & 0 & 1 \\
0 & 5 & 1 \pm \omega_{21} \\
1 & 1 \pm \bar{\omega}_{21} & 5
\end{array}\right), \\
\langle 1\rangle \perp\left(\begin{array}{ccc}
2 & 0 & 1 \\
0 & 5 & 2 \pm \omega_{33} \\
1 & 2 \pm \bar{\omega}_{33} & 8
\end{array}\right), & \langle 1\rangle \perp\left(\begin{array}{ccc}
2 & 0 & 1 \\
0 & 5 & 1 \pm \omega_{41} \\
1 & 1 \pm \bar{\omega}_{41} & 9
\end{array}\right) .
\end{array}
$$

If $\ell$ does not represent 15 , then we can obtain a universal pro forma quinary Hermitian lattice by attaching a vector of norm 15 to $\ell$. If $\ell$ is inherited and it is not 
universal, then $\ell$ is one of the following 4 lattices whose truants are all 15:

$$
\langle 1\rangle \perp\left(\begin{array}{lll}
2 & 0 & 0 \\
0 & 5 & 0 \\
0 & 0 & 5
\end{array}\right),\langle 1\rangle \perp\left(\begin{array}{lll}
2 & 0 & 1 \\
0 & 5 & 1 \\
1 & 1 & 5
\end{array}\right),\langle 1\rangle \perp\left(\begin{array}{lll}
2 & 0 & 1 \\
0 & 5 & 2 \\
1 & 2 & 8
\end{array}\right),\langle 1\rangle \perp\left(\begin{array}{lll}
2 & 0 & 1 \\
0 & 5 & 1 \\
1 & 1 & 9
\end{array}\right) .
$$

In this case, we can obtain a universal lattice by attaching a vector of norm 15 . Note that these quinary universal lattices are inherited if $m \geq 129$.

Case III-4 b(2). $m \equiv 3(\bmod 4)$ : From the positive semi-definiteness, the escalation lattices $\ell$ are all inherited for $m \geq 203$. We may assume that $m=$ $35,43,47,51, \ldots, 199$. Note that $\langle 1,2,5\rangle_{\mathbb{Z}}$ represents all positive integers except the form $5^{d} u_{-}$. If $n=5^{d} u_{-} \geq 3 m$, then at least one of $n-m, n-2 m$ and $n-3 m$ is not of the form $5^{d} u_{-}$. Thus $n \rightarrow\langle 1,2,5\rangle$. If $\ell$ represents all positive integers $n<3 m$, then $\ell$ is universal. If $\ell$ is not universal and uninherited, then $\ell$ is one of the following and their conjugates:

$$
\begin{array}{ccc}
\langle 1\rangle \perp\left(\begin{array}{ccc}
2 & 0 & 0 \\
0 & 5 & 1+\omega \\
0 & 1+\bar{\omega} & 8
\end{array}\right) & \text { if } m=47,55,151,67 \leq m \leq 131, \\
\langle 1\rangle \perp\left(\begin{array}{ccc}
2 & 0 & 0 \\
0 & 5 & -2+\omega \\
0 & -2+\bar{\omega} & 8
\end{array}\right) & \text { if } m=47,55,151,67 \leq m \leq 131, \\
\langle 1\rangle \perp\left(\begin{array}{ccc}
2 & 0 & 0 \\
0 & 5 & 2+\omega \\
0 & 2+\bar{\omega} & 8 \\
2 & 0 & 1 \\
0 & 5 & 2+\omega \\
1 & 2+\bar{\omega} & 9 \\
2 & 0 & 0 \\
0 & 5 & 2+\omega \\
0 & 2+\bar{\omega} & 10
\end{array}\right) & \text { if } m=47,55,67 \leq m \leq 119, \\
\langle 1\rangle \perp & \text { if } m=47,55,67 \leq m \leq 131, \\
\langle 1\rangle \perp m=47,55,67 \leq m \leq 159 .
\end{array}
$$

We can check that $\ell$ represents all positive integers except only 15 . Hence we can obtain a universal lattice by attaching a vector of norm 15 to $\ell$. If $\ell$ is inherited and $\ell$ is not universal, then we also obtain a universal lattice by the same process. Case III-5. $\langle 1\rangle \perp\left(\begin{array}{ll}2 & 1 \\ 1 & 4\end{array}\right) \rightarrow L$ : Note that $\langle 1\rangle \perp\left(\begin{array}{ll}2 & 1 \\ 1 & 4\end{array}\right)$ represents a quadratic lattice $\langle 1\rangle_{\mathbb{Z}} \perp\left(\begin{array}{ll}2 & 1 \\ 1 & 4\end{array}\right)_{\mathbb{Z}}$ whose truant is 7 .

Case III-5 a. $7 \rightarrow\langle 1\rangle \perp\left(\begin{array}{ll}2 & 1 \\ 1 & 4\end{array}\right)$ : Then we only have $m=6$. Since $\langle 1\rangle \perp$ $\left(\begin{array}{ll}2 & 1 \\ 1 & 4\end{array}\right)$ represents a universal quadratic lattice $\langle 1,6\rangle_{\mathbb{Z}} \perp\left(\begin{array}{ll}2 & 1 \\ 1 & 4\end{array}\right)_{\mathbb{Z}},\langle 1\rangle \perp\left(\begin{array}{ll}2 & 1 \\ 1 & 4\end{array}\right)$ is universal. From now through the Case III-5, we may assume that $m \neq 6$.

Case III-5 b. $7 \nrightarrow \rightarrow\langle 1\rangle \perp\left(\begin{array}{ll}2 & 1 \\ 1 & 4\end{array}\right)$ : The next escalation lattice $\ell$ of $\langle 1\rangle \perp\left(\begin{array}{ll}2 & 1 \\ 1 & 4\end{array}\right)$ is of the form

$$
\ell \cong\left(\begin{array}{cccc}
1 & 0 & 0 & \alpha \\
0 & 2 & 1 & \beta \\
0 & 1 & 4 & \gamma \\
\bar{\alpha} & \bar{\beta} & \bar{\gamma} & 7
\end{array}\right)
$$

for some $\alpha, \beta$ and $\gamma$. 
Case III-5 $\mathbf{b}(\mathbf{1}) \cdot m \not \equiv 3(\bmod 4)$ : From the positive semi-definiteness, $\ell$ is inherited if $m \geq 29$, and $\ell$ represents a quadratic lattice

$$
\left(\begin{array}{cccc}
1 & 0 & 0 & \operatorname{Re} \alpha \\
0 & 2 & 1 & \operatorname{Re} \beta \\
0 & 1 & 4 & \operatorname{Re} \gamma \\
\operatorname{Re} \alpha & \operatorname{Re} \beta & \operatorname{Re} \gamma & 7
\end{array}\right)_{\mathbb{Z}}
$$

There are 30 quadratic lattices of this type up to isometry. These quadratic lattices are universal except for the following lattice:

$$
\ell^{\prime}=\langle 1\rangle_{\mathbb{Z}} \perp\left(\begin{array}{lll}
2 & 1 & 0 \\
1 & 4 & 1 \\
0 & 1 & 5
\end{array}\right)_{\mathbb{Z}}
$$

Note that the truant of $\ell^{\prime}$ is 10 and $\ell^{\prime}$ represents all numbers 1 to 15 except 10 . Assume that $\ell$ represents $\ell^{\prime}$. If $m=10$, then the Hermitian lattice $\ell$ represents 10 ; hence $\ell$ is a quaternary universal Hermitian lattice. From the positive semidefiniteness, if $m \geq 17$, then $\ell$ is inherited. If $m=13,14$ and $\ell$ is uninherited, then

$$
\ell \cong\langle 1\rangle \perp\left(\begin{array}{ccc}
2 & 1 & 0 \\
1 & 4 & 1 \pm \omega_{m} \\
0 & 1 \pm \bar{\omega}_{m} & 5
\end{array}\right)
$$

Since $\ell$ represents all positive integers 1 through 15 except only the truant 10 of $\ell$, the next escalation lattice of $\ell$ is a pro forma quinary universal Hermitian lattice which can be obtained by attaching a vector of norm 10 . In this case, the universal Hermitian lattice is inherited if $m \geq 53$. If $\ell$ is inherited and it is not universal, then $\ell$ is the following lattice whose truant is 10 :

$$
\langle 1\rangle \perp\left(\begin{array}{lll}
2 & 1 & 0 \\
1 & 4 & 1 \\
0 & 1 & 5
\end{array}\right) .
$$

Hence we can obtain a universal lattice via attaching a vector of norm 10 .

Case III-5 b(2). $m \equiv 3(\bmod 4)$ : From the positive semi-definiteness, the escalation lattices $\ell$ are all inherited for $m \geq 115$. Thus we may assume that $m=23,31,35, \ldots, 111$. Note that $\langle 1\rangle_{\mathbb{Z}} \perp\left(\begin{array}{ll}2 & 1 \\ 1 & 4\end{array}\right)_{\mathbb{Z}}$ represents positive integers $n$ when $n \neq 7^{d} u_{-}$and $n \not \equiv 7,10(\bmod 12)$. The lattice $\langle 1\rangle \perp\left(\begin{array}{ll}2 & 1 \\ 1 & 4\end{array}\right)$ represents $\langle 1\rangle_{\mathbb{Z}} \perp\left(\begin{array}{ll}2 & 1 \\ 1 & 4\end{array}\right)_{\mathbb{Z}}+k m$ with $k=1,2,3$. If $n$ is not represented by $\langle 1\rangle_{\mathbb{Z}} \perp\left(\begin{array}{ll}2 & 1 \\ 1 & 4\end{array}\right)_{\mathbb{Z}}$, then at least one of $n-m, n-2 m$ and $n-3 m$ is represented by $\langle 1\rangle_{\mathbb{Z}} \perp\left(\begin{array}{ll}2 & 1 \\ 1 & 4\end{array}\right)_{\mathbb{Z}}$ for $n \geq 3 \mathrm{~m}$. It can be verified that $\ell$ represents all positive integers smaller than $3 m$. Hence the universality of $\ell$ is proved. If $m \geq 115$, then all $\ell$ 's are universal except $\langle 1\rangle \perp\left(\begin{array}{lll}2 & 1 & 0 \\ 1 & 4 & 1 \\ 0 & 1 & 5\end{array}\right)$. We can obtain a universal pro forma quinary lattice by attaching a vector of norm 10 to this exception. 
Case III-6. $\langle 1\rangle \perp\left(\begin{array}{ll}2 & 1 \\ 1 & 5\end{array}\right) \rightarrow L$ : Note that $\langle 1\rangle \perp\left(\begin{array}{ll}2 & 1 \\ 1 & 5\end{array}\right)$ represents a quadratic lattice $\langle 1\rangle_{\mathbb{Z}} \perp\left(\begin{array}{ll}2 & 1 \\ 1 & 5\end{array}\right)_{\mathbb{Z}}$ whose truant is 7 .

Case III-6 a. $7 \rightarrow\langle 1\rangle \perp\left(\begin{array}{ll}2 & 1 \\ 1 & 5\end{array}\right)$ : We have only $m=6$. Since $\langle 1\rangle \perp\left(\begin{array}{ll}2 & 1 \\ 1 & 5\end{array}\right)$ represents a universal quadratic lattice $\langle 1,6\rangle_{\mathbb{Z}} \perp\left(\begin{array}{ll}2 & 1 \\ 1 & 5\end{array}\right)_{\mathbb{Z}},\langle 1\rangle \perp\left(\begin{array}{ll}2 & 1 \\ 1 & 5\end{array}\right)$ is universal. From now through the Case III-6, we may assume that $m \neq 6$.

Case III-6 b. $7 \nrightarrow \rightarrow\langle 1\rangle \perp\left(\begin{array}{ll}2 & 1 \\ 1 & 5\end{array}\right)$ : The next escalation lattice $\ell$ of $\langle 1\rangle \perp\left(\begin{array}{ll}2 & 1 \\ 1 & 5\end{array}\right)$ is of the form

$$
\ell \cong\left(\begin{array}{cccc}
1 & 0 & 0 & \alpha \\
0 & 2 & 1 & \beta \\
0 & 1 & 5 & \gamma \\
\bar{\alpha} & \bar{\beta} & \bar{\gamma} & 7
\end{array}\right)
$$

for some $\alpha, \beta, \gamma$.

Case III-6 b(1). $m \not \equiv 3(\bmod 4)$ : From the positive semi-definiteness, $\ell$ is inherited if $m \geq 37$, and $\ell$ represents a quadratic lattice

$$
\left(\begin{array}{cccc}
1 & 0 & 0 & \operatorname{Re} \alpha \\
0 & 2 & 1 & \operatorname{Re} \beta \\
0 & 1 & 5 & \operatorname{Re} \gamma \\
\operatorname{Re} \alpha & \operatorname{Re} \beta & \operatorname{Re} \gamma & 7
\end{array}\right)_{\mathbb{Z}} .
$$

There are 16 quadratic lattices of this type up to isometry. These quadratic lattices are universal except for the following lattice:

$$
\ell^{\prime}=\langle 1\rangle_{\mathbb{Z}} \perp\left(\begin{array}{lll}
2 & 1 & 0 \\
1 & 5 & 1 \\
0 & 1 & 5
\end{array}\right)_{\mathbb{Z}} .
$$

Note that the truant of $\ell^{\prime}$ is 15 and $\ell^{\prime}$ represents all numbers 1 to 14 . Assume $\ell$ represents $\ell^{\prime}$. If $\ell$ is uninherited, then $m=10,13,14,17,21$ and

$$
\ell \cong\langle 1\rangle \perp\left(\begin{array}{ccc}
2 & 1 & 0 \\
1 & 5 & 1 \pm \omega_{m} \\
0 & 1 \pm \bar{\omega}_{m} & 5
\end{array}\right)
$$

Note that if $m=10,13,14,21$, then $\ell$ represents 15 . Hence $\ell$ is a quaternary universal lattice. If $m=17$, then it is isometric to the lattice in the Case III- $4 \mathrm{~b}(1)$. If $\ell$ is inherited, and it is not universal, then $\ell$ is the following lattice whose truant is 15 :

$$
\langle 1\rangle \perp\left(\begin{array}{lll}
2 & 1 & 0 \\
1 & 5 & 1 \\
0 & 1 & 5
\end{array}\right) .
$$

Thus we can obtain a universal lattice via attaching a vector of norm 15 .

Case III-6 $\mathbf{b}(\mathbf{2}) . \quad m \equiv 3(\bmod 4)$ : From the positive semi-definiteness, the escalation lattices $\ell$ are inherited for $m \geq 147$. Thus we may assume that $m=$ 
$23,31,35, \ldots, 139$. Note that $\langle 1\rangle_{\mathbb{Z}} \perp\left(\begin{array}{ll}2 & 1 \\ 1 & 5\end{array}\right)_{\mathbb{Z}}$ represents all positive integers $n$ except the form $2^{e} u_{7}$. The associated (nonclassical) quadratic lattice of $\langle 1\rangle \perp\left(\begin{array}{ll}2 & 1 \\ 1 & 5\end{array}\right)$ represents $\langle 1\rangle_{\mathbb{Z}} \perp\left(\begin{array}{ll}2 & 1 \\ 1 & 5\end{array}\right)_{\mathbb{Z}} \perp\langle m\rangle_{\mathbb{Z}}$. If $n=2^{e} u_{7}>4 m$ is not represented by $\langle 1\rangle_{\mathbb{Z}} \perp\left(\begin{array}{ll}2 & 1 \\ 1 & 5\end{array}\right)_{\mathbb{Z}}$, then at least one of $n-m$ and $n-4 m$ is represented by $\langle 1\rangle_{\mathbb{Z}} \perp\left(\begin{array}{ll}2 & 1 \\ 1 & 5\end{array}\right)_{\mathbb{Z}}$. It is also verified that each $\ell$ represents all positive integers smaller than $4 m$. Hence the universality of the $\ell$ 's is proved. If $m \geq 147$, then all the $\ell$ 's are universal except $\langle 1\rangle \perp\left(\begin{array}{lll}2 & 1 & 0 \\ 1 & 5 & 1 \\ 0 & 1 & 5\end{array}\right)$. We can obtain a universal pro forma quinary lattice by attaching a vector of norm 15 to this exception.

Case III-7. Now we investigate the lattices

$$
\langle 1\rangle \perp\left(\begin{array}{cc}
2 & \omega \\
\bar{\omega} & b
\end{array}\right) \text { and }\langle 1\rangle \perp\left(\begin{array}{cc}
2 & -1+\omega \\
-1+\bar{\omega} & b
\end{array}\right)
$$

over $\mathbb{Q}(\sqrt{-m})$ in Table 1, It is known that all the binary Hermitian lattices in Table 1 are universal (see [10]) except for the last two over $\mathbb{Q}(\sqrt{-39})$ and they are listed at the end of this paper (see Table 3). If $m \neq \equiv 3(\bmod 4)$, i.e., $m=6,10$, then the universalities of ternary lattices in Table 1 are checked by the fifteen theorem. Now assume that $m \equiv 3(\bmod 4)$, i.e., $m=15,23,31,35,39$. Since

$$
\langle 1\rangle \perp\left(\begin{array}{cc}
2 & -1+\omega \\
-1+\bar{\omega} & b
\end{array}\right)=\overline{\langle 1\rangle \perp\left(\begin{array}{cc}
2 & \omega \\
\bar{\omega} & b
\end{array}\right)},
$$

it is enough to check the universality of $\ell \cong\langle 1\rangle \perp\left(\begin{array}{cc}2 & \omega \\ \bar{\omega} & b\end{array}\right)$. Each associated quadratic lattice of $\ell$ has a sublattice $\ell^{\prime}$ of class number 1 (see Table 2). The universality of each lattice $\ell^{\prime}$ is proved by a method similar to the previous case.

The last exceptional lattice is

$$
\ell \cong\langle 1\rangle \perp\left(\begin{array}{cc}
2 & \omega \\
\bar{\omega} & 5
\end{array}\right)
$$

over $\mathbb{Q}(\sqrt{-39})$. The associated quadratic form of this lattice is

$$
N: x_{1}^{2}+x_{1} x_{2}+10 x_{2}^{2}+2 y_{1}^{2}+y_{1} z_{1}+5 z_{1}^{2} .
$$

Since $2 N \rightarrow N$, we only need to show that $N$ represents all odd positive integers. If we set $x_{2}=2 t$ for some $t \in \mathbb{Z}$, then $N$ represents

$$
\langle 1\rangle_{\mathbb{Z}} \perp\left(\begin{array}{cc}
2 & 1 / 2 \\
1 / 2 & 5
\end{array}\right)_{\mathbb{Z}} \perp\langle 39\rangle_{\mathbb{Z}}
$$

For sufficiently large $n$, we will show that

$$
n-39 s^{2} \rightarrow N^{\prime}=\langle 1\rangle_{\mathbb{Z}} \perp\left(\begin{array}{cc}
2 & 1 / 2 \\
1 / 2 & 5
\end{array}\right)_{\mathbb{Z}}
$$


TABLe 2. Sublattices of class number 1

\begin{tabular}{|c|c|c|c|c|c|c|c|c|c|c|c|c|c|}
\hline field & \multicolumn{12}{|c|}{ lattice } & subtrahend \\
\hline \multirow{9}{*}{$\mathbb{Q}(\sqrt{-15})$} & 71 & 0 & & & 71 & 0 & $0)$ & & $\overline{71}$ & 0 & $0)$ & \multirow[b]{2}{*}{$\leftarrow n \neq 2^{d} u_{7}$} & \multirow{3}{*}{15} \\
\hline & & 2 & $\omega$ & $\leftarrow$ & 0 & 2 & 4 & $\cong$ & 0 & 2 & 0 & & \\
\hline & ( & $\bar{\omega}$ & 3) & & 0 & 4 & 12) & & 0 & 0 & 4) & & \\
\hline & i & 0 & $0)$ & & $(1$ & 0 & $0)$ & & 1 & 0 & $0)$ & & \multirow{3}{*}{$15,2 \cdot 15$} \\
\hline & & 2 & $\omega$ & $\leftarrow$ & 0 & 2 & 4 & $\cong$ & 0 & 2 & 0 & \multirow{2}{*}{$\leftarrow n \neq u_{5}, 2^{e} u_{7}$} & \\
\hline & ( & $\bar{\omega}$ & & & 0 & 4 & 16) & & 10 & 0 & $8)$ & & \\
\hline & 1 & 0 & & & 1 & 0 & $0)$ & & 1 & 0 & $0)$ & & \\
\hline & & 2 & $\omega$ & $\leftarrow$ & 0 & 8 & 4 & & 0 & 5 & 1 & $\leftarrow n \neq 2^{d} u_{5}$ & \multirow[t]{2}{*}{15} \\
\hline & ( & $\bar{\omega}$ & 5) & & 0 & 4 & 5) & & 0 & 1 & 5) & & \\
\hline \multirow{6}{*}{$\mathbb{Q}(\sqrt{-23})$} & 1 & 0 & $0)$ & & 1 & 0 & $0)$ & & 71 & 0 & $0\rangle$ & \multirow{3}{*}{$\leftarrow n \neq 2^{e} u_{5}$} & \multirow{3}{*}{23} \\
\hline & & 2 & $\omega$ & $\leftarrow$ & 0 & 2 & 6 & $\cong$ & 0 & 2 & 0 & & \\
\hline & ( & $\bar{\omega}$ & 4) & & 0 & 6 & 24) & & 0 & 0 & 6 ) & & \\
\hline & 1 & 0 & $0)$ & & 1 & 0 & $0)$ & & 1 & 0 & $0)$ & \multirow{3}{*}{$\leftarrow n \neq 2^{d} u_{5}$} & \multirow{3}{*}{23} \\
\hline & & 2 & $\omega$ & $\leftarrow$ & 0 & 12 & 6 & $\cong$ & 0 & 5 & 1 & & \\
\hline & ( & $\bar{\omega}$ & 5) & & 0 & 6 & $5)$ & & 0 & 1 & 5) & & \\
\hline \multirow{3}{*}{$\mathbb{Q}(\sqrt{-31})$} & 7 & 0 & $0)$ & & 71 & 0 & $0)$ & & 71 & $\overline{0}$ & $0)$ & \multirow{3}{*}{$\leftarrow n \neq u_{5}, 2^{e} u_{7}$} & \multirow{3}{*}{$15,2 \cdot 15$} \\
\hline & & 2 & $\omega$ & $\leftarrow$ & 0 & 2 & 8 & $\cong$ & 0 & 2 & 0 & & \\
\hline & ( & $\bar{\omega}$ & 5) & & 0 & 8 & $40)$ & $\mathbb{Z}$ & 0 & 0 & $8)$ & & \\
\hline \multirow{3}{*}{$\mathbb{Q}(\sqrt{-35})$} & 7 & $\overline{0}$ & $0)$ & & 71 & 0 & $0)$ & & 1 & $\overline{0}$ & $0)$ & \multirow{3}{*}{$\leftarrow n \neq 2^{e} u_{7}$} & \multirow{3}{*}{$35,2^{2} \cdot 35$} \\
\hline & ( & 2 & $\omega$ & $\leftarrow$ & 0 & 2 & 9 & $\cong$ & 0 & 2 & 1 & & \\
\hline & lo & $\bar{\omega}$ & 5 & & 0 & 9 & 45) & 7 & (c) & 1 & 5 & & \\
\hline
\end{tabular}

for suitable $s$. This implies the desired result: $n \rightarrow \ell$ over $\mathbb{Q}(\sqrt{-39})$. Note that $N^{\prime}$ represents two quadratic sublattices which are in the same genus by the BrandtIntrau table [2]:

$$
\left(\begin{array}{ccc}
1 & 0 & 0 \\
0 & 8 & 2 \\
0 & 2 & 20
\end{array}\right)_{\mathbb{Z}},\left(\begin{array}{lll}
4 & 0 & 2 \\
0 & 5 & 1 \\
2 & 1 & 9
\end{array}\right)_{\mathbb{Z}} .
$$

Hence if we show that $n-39 s^{2}$ is represented by the genus, then we can say $n \rightarrow \ell$. We have that the genus represents all positive integers $n$ when $n \equiv 0,1(\bmod 4)$ and $n \neq 13^{d} u_{+}$. For other odd integers $n \geq 39 \cdot 6^{2}$, here are choices for $s$ as follows:

\begin{tabular}{c|l|l}
\hline$n$ & \multicolumn{1}{|c}{$u_{ \pm}$} & subtrahend \\
\hline$n \equiv 1(\bmod 4), n=13^{d} u_{+}$ & $u_{+} \equiv 1,4,10(\bmod 13)$ & $39 \cdot 2^{2}$ \\
& $u_{+} \equiv 3(\bmod 13)$ & $39 \cdot 4^{2}$ \\
& $u_{+} \equiv 9,12(\bmod 13)$ & $39 \cdot 6^{2}$ \\
\hline$n \equiv 3(\bmod 4), n=13^{e} u_{+}$ & $u_{+} \equiv 1,4,9,10,12(\bmod 13)$ & $39 \cdot 1^{2}$ \\
& $u_{+} \equiv 3(\bmod 13)$ & $39 \cdot 3^{2}$ \\
\hline$n \equiv 3(\bmod 4), n=13^{e} u_{-}$ & $u_{-} \equiv 2,5,6,7,8,11(\bmod 13)$ & $39 \cdot 1^{2}$ \\
\hline$n \equiv 3(\bmod 4), n=13^{d} u_{+}$ & $u_{+} \equiv 1,9,10(\bmod 13)$ & $39 \cdot 1^{2}$ \\
& $u_{+} \equiv 3,12(\bmod 13)$ & $39 \cdot 3^{2}$ \\
& $u_{+} \equiv 4(\bmod 13)$ & $39 \cdot 5^{2}$ \\
\hline$n \equiv 3(\bmod 4), n=13^{d} u_{-}$ & $u_{-} \equiv 2(\bmod 13)$ & $39 \cdot 5^{2}$ \\
& $u_{-} \equiv 5,8,11(\bmod 13)$ & $39 \cdot 1^{2}$ \\
& $u_{-} \equiv 6,7(\bmod 13)$ & $39 \cdot 3^{2}$ \\
\hline
\end{tabular}


TABLe 3. Binary universal Hermitian lattice

\begin{tabular}{lll}
\hline $\mathbb{Q}(\sqrt{-m})$ & binary universal lattices \\
\hline $\mathbb{Q}(\sqrt{-1})$ & $\langle 1,1\rangle,\langle 1,2\rangle,\langle 1,3\rangle$ \\
$\mathbb{Q}(\sqrt{-2})$ & $\langle 1,1\rangle,\langle 1,2\rangle,\langle 1,3\rangle,\langle 1,4\rangle,\langle 1,5\rangle$ \\
$\mathbb{Q}(\sqrt{-3})$ & $\langle 1,1\rangle,\langle 1,2\rangle$ \\
$\mathbb{Q}(\sqrt{-5})$ & $\langle 1,2\rangle,\langle 1\rangle \perp\left(\begin{array}{cc}2 & -1+\omega_{5} \\
-1+\bar{\omega}_{5} & 3\end{array}\right)$ \\
$\mathbb{Q}(\sqrt{-6})$ & $\langle 1\rangle \perp\left(\begin{array}{cc}2 & \omega_{6} \\
\bar{\omega}_{6} & 3\end{array}\right)$ \\
$\mathbb{Q}(\sqrt{-7})$ & $\langle 1,1\rangle,\langle 1,2\rangle,\langle 1,3\rangle$ \\
$\mathbb{Q}(\sqrt{-10})$ & $\langle 1\rangle \perp\left(\begin{array}{cc}2 & \omega_{10} \\
\bar{\omega}_{10} & 5\end{array}\right)$ \\
$\mathbb{Q}(\sqrt{-11})$ & $\langle 1,1\rangle,\langle 1,2\rangle$ \\
$\mathbb{Q}(\sqrt{-15})$ & $\langle 1\rangle \perp\left(\begin{array}{cc}2 & \omega_{15} \\
\bar{\omega}_{15} & 2\end{array}\right)$ \\
$\mathbb{Q}(\sqrt{-19})$ & $\langle 1,2\rangle$ & \\
& \\
$\mathbb{Q}(\sqrt{-23})$ & $\langle 1\rangle \perp\left(\begin{array}{cc}2 & \omega_{23} \\
\omega_{23} & 3\end{array}\right),\langle 1\rangle \perp\left(\begin{array}{cc}2 & -1+\omega_{23} \\
-1+\bar{\omega}_{23} & 3 \\
2 & -1+\omega_{31} \\
2 & \omega_{31} \\
\omega_{31} & 4\end{array}\right),\langle 1\rangle \perp\left(\begin{array}{cc} \\
-1+\bar{\omega}_{31} & 4\end{array}\right)$ \\
$\mathbb{Q}(\sqrt{-31})$ & $\langle 1\rangle \perp$ \\
\hline
\end{tabular}

For $n<39 \cdot 6^{2}$, we can check that $\ell$ represents all integers $n$ except 13 and 91 by direct calculation. If we attach a vector of norm 13 to $\ell$, the escalation lattices also represent 91 . Hence they are universal Hermitian lattices.

Remark 1. In this article, we computed all new universal Hermitian lattices which do not contain other universal lattices. For example, over $\mathbb{Q}(\sqrt{-5})$, there are 2 binary universal Hermitian lattices as follows, up to isometry [10]:

$$
\langle 1,2\rangle,\langle 1\rangle \perp\left(\begin{array}{cc}
2 & -1+\omega \\
-1+\bar{\omega} & 3
\end{array}\right) .
$$

The first lattice is found in the procedure of escalation in this article, but the second one is excluded because it contain a universal Hermitian lattice $\langle 1,2\rangle$ as a sublattice.

Remark 2. We computed all quaternary escalation lattices and corresponding truants (see Table 4). We can obtain pro forma quinary universal Hermitian lattices by attaching a vector whose norm is its truant to each quaternary escalation lattice. But these are too numerous to list them all. 
TABLE 4. Truants of escalation lattices

\begin{tabular}{|c|c|c|c|c|}
\hline \multicolumn{3}{|c|}{ Escalation lattice } & Truant & $m$ \\
\hline \multirow{2}{*}{\multicolumn{3}{|c|}{$\langle 1\rangle$}} & 2 & if $m \neq 1,2 ; 7$ \\
\hline & & & $\begin{array}{l}3 \\
5\end{array}$ & $\begin{array}{l}\text { if } m=1 ; 7 \\
\text { if } m=2\end{array}$ \\
\hline \multicolumn{3}{|l|}{$\langle 1,1\rangle$} & 3 & if $m \neq 1,2 ; 3,7,11$ \\
\hline \multirow{2}{*}{\multicolumn{3}{|c|}{$\langle 1,1,1\rangle$}} & 7 & if $m \neq 1,2,5,6 ; 3,7,11,15,19,23$, \\
\hline & & & 14 & if $m \neq 1,2,5,6,10,13,14 ; 3,7,11,15,19,23,31,35,39,43,47,51,55$, \\
\hline \multicolumn{3}{|l|}{$\langle 1,1,3\rangle$} & 6 & if $m \neq 1,2,5,6 ; 3,7,11,15,19,23$ \\
\hline \multicolumn{3}{|l|}{$\begin{array}{l}1,1,3\rangle \\
\langle 1,2\rangle\end{array}$} & 5 & if $m \neq 1,2,5 ; 3,7,11,19$ \\
\hline \multirow{2}{*}{\multicolumn{3}{|c|}{$\langle 1,2,2\rangle$}} & 7 & if $m \neq 1,2,5,6 ; 3,7,11,15,19$ \\
\hline \multirow{2}{*}{\multicolumn{3}{|c|}{$\begin{array}{l}\langle 1,2,3\rangle \\
\langle 1,2,4\rangle\end{array}$}} & 10 & if $m \neq 1,2,5,6,10 ; 3,7,11,15,19,23,31,39$ \\
\hline & & & 14 & if $m \neq 1,2,5,6,10,13,14 ; 3,7,11,15,19,23,31,39,47,55$, \\
\hline & 10 & if $m \neq 1,2,5,6,10 ; 3,7,11,15,19,23,31,39$ \\
\hline \multirow{2}{*}{$\langle 1\rangle \perp\left(\begin{array}{l}2 \\
0 \\
0\end{array}\right.$} & $0)$ & & & if $m \equiv 1,2(\bmod 4)$ and $m \geq 22$ \\
\hline & $\left.\begin{array}{ll}5 & 0 \\
0 & 5\end{array}\right)$ & & 15 & if $m \equiv 3(\bmod 4)$ and $m=47,55, m \geq 67$ \\
\hline 2 & $1)$ & & & if $m \equiv 1,2(\bmod 4)$ and $m \geq 21$ \\
\hline \multirow{2}{*}{$\langle 1\rangle \perp\left(\begin{array}{l}2 \\
0 \\
1 \\
2\end{array}\right.$} & $\left.\begin{array}{l}1 \\
5\end{array}\right)$ & & 15 & if $m \equiv 3(\bmod 4)$ and $m=47,55, m \geq 67$ \\
\hline & $1\}$ & & & if $m \equiv 1,2(\bmod 4)$ and $m \geq 33$ \\
\hline \multirow{2}{*}{$\langle 1\rangle \perp \mid \begin{array}{l}0 \\
1 \\
2\end{array}$} & $\left.\begin{array}{l}2 \\
8\end{array}\right)$ & & 15 & if $m \equiv 3(\bmod 4)$ and $m=\overline{4} 7,55, m \geq 67$ \\
\hline & $1)$ & & & if $m \equiv 1,2(\bmod 4)$ and $m \geq 41$ \\
\hline$\langle 1\rangle \perp\left(\begin{array}{l}0 \\
1\end{array}\right.$ & $\left.\begin{array}{ll}5 & 1 \\
1 & 9\end{array}\right)$ & & 15 & if $m \equiv 3(\bmod 4)$ and $m=47,55, m \geq 67$, \\
\hline$\langle 1\rangle \perp\left(\begin{array}{l}2 \\
0 \\
0\end{array}\right.$ & $\begin{array}{c}0 \\
5 \\
\pm \bar{\omega}\end{array}$ & $\left.\begin{array}{l}0 \\
\pm \omega \\
5\end{array}\right)$ & 15 & if $m=17,21$ \\
\hline$\langle 1\rangle \perp\left(\begin{array}{l}2 \\
0 \\
1 \\
2\end{array}\right.$ & $\begin{array}{l}0 \\
5 \\
1 \pm \varpi\end{array}$ & $\left.\begin{array}{c}1 \\
1 \pm \omega \\
5\end{array}\right)$ & 15 & if $m=17$, \\
\hline 2 & & $1\}$ & & \\
\hline$\langle 1\rangle \perp\left(\begin{array}{l}2 \\
0 \\
1\end{array}\right.$ & $\begin{array}{l}5 \\
2 \pm \bar{\omega}\end{array}$ & $2 \pm \omega$ & 15 & if $m=17,21,22,26,29,30$, \\
\hline \multirow{2}{*}{$\langle 1\rangle \perp\left(\begin{array}{l}2 \\
0 \\
1\end{array}\right.$} & $\begin{array}{l}0 \\
5\end{array}$ & $\begin{array}{c}1 \\
1 \pm \omega\end{array}$ & 15 & if $m=17,21,22,26,29,30,33,34,37,38$ \\
\hline & $\begin{array}{l}1 \pm \bar{\omega} \\
0\end{array}$ & $\begin{array}{l}9 \\
0\end{array}$ & & \\
\hline$\langle 1\rangle \perp\left(\begin{array}{l}2 \\
0 \\
0 \\
2\end{array}\right.$ & $\begin{array}{c}5 \\
1+\bar{\omega}\end{array}$ & $\left.\begin{array}{c}1+\omega \\
8\end{array}\right)$ & 15 & if $m \equiv 3(\bmod 4)$ and $m=47,55,151,67 \leq m \leq 131$ \\
\hline $\mid \begin{array}{l}0 \\
0 \\
2\end{array}$ & $\begin{array}{c}0 \\
5 \\
-2+\bar{\omega} \\
0\end{array}$ & $\left.\begin{array}{c}0 \\
-2+\omega \\
8 \\
0\end{array}\right)$ & 15 & if $m \equiv 3(\bmod 4)$ and $m=47,55,151,67 \leq m \leq 131$ \\
\hline$\langle 1\rangle \perp\left(\begin{array}{l}0 \\
0 \\
0\end{array}\right.$ & $\begin{array}{c}5 \\
2+\bar{\omega}\end{array}$ & $\begin{array}{c}2+\omega \\
8\end{array}$ & 15 & if $m \equiv 3(\bmod 4)$ and $m=47,55,67 \leq m \leq 119$ \\
\hline$\langle 1\rangle \perp\left(\begin{array}{l}2 \\
0\end{array}\right.$ & $\begin{array}{c}0 \\
5 \\
2+\varpi\end{array}$ & $\left.\begin{array}{c}1 \\
2+\omega \\
9\end{array}\right)$ & 15 & if $m \equiv 3(\bmod 4)$ and $m=47,55,67 \leq m \leq 131$ \\
\hline$\langle 1\rangle \perp\left(\begin{array}{l}2 \\
0 \\
0\end{array}\right.$ & $\begin{array}{c}0 \\
5 \\
2+\bar{\omega}\end{array}$ & $\left.\begin{array}{c}0 \\
2+\omega \\
10\end{array}\right)$ & 15 & if $m \equiv 3(\bmod 4)$ and $m=47,55,67 \leq m \leq 159$ \\
\hline$\langle 1\rangle \perp\left(\begin{array}{l}2 \\
1\end{array}\right.$ & $\left.\begin{array}{l}1 \\
4\end{array}\right)$ & & 7 & if $m \neq 1,2,5,6,10 ; 3,7,11,19$ \\
\hline$\langle 1\rangle \perp\left(\begin{array}{l}2 \\
1\end{array}\right.$ & $\left.\begin{array}{ll}1 & 0 \\
4 & 1 \\
1 & 5\end{array}\right)$ & & 10 & $\begin{array}{l}\text { if } m \equiv 1,2(\bmod 4) \text { and } m \geq 17, \\
\text { if } m \equiv 3(\bmod 4) \text { and } m \geq 115 \text {, }\end{array}$ \\
\hline$\langle 1\rangle \perp\left(\begin{array}{l}2 \\
1 \\
0\end{array}\right)$ & $\begin{array}{c}1 \\
4 \\
1 \pm \varpi\end{array}$ & $\left.\begin{array}{c}0 \\
1 \pm \omega \\
5\end{array}\right)$ & 10 & if $m=13,14$ \\
\hline$\langle 1\rangle \perp\left(\begin{array}{l}2 \\
1\end{array}\right.$ & $\left.\begin{array}{l}1 \\
5\end{array}\right)$ & & 7 & if $m \neq 1,2,5,6 ; 3,7,11,19$ \\
\hline$\langle 1\rangle \perp\left(\begin{array}{l}2 \\
1 \\
0\end{array}\right.$ & $\left.\begin{array}{ll}1 & 0 \\
5 & 1 \\
1 & 5\end{array}\right)$ & & 15 & $\begin{array}{l}\text { if } m \equiv 1,2(\bmod 4) \text { and } m \geq 21, \\
\text { if } m \equiv 3(\bmod 4) \text { and } m \geq 147 \text {, }\end{array}$ \\
\hline$\langle 1\rangle \perp\left(\begin{array}{l}2 \\
1 \\
0\end{array}\right)$ & $\begin{array}{c}1 \\
5 \\
1 \pm \bar{\omega}\end{array}$ & $\left.\begin{array}{c}0 \\
1 \pm \omega \\
5\end{array}\right)$ & 15 & if $m=17$, \\
\hline$\langle 1\rangle \perp\left(\frac{2}{\omega}\right.$ & $\left.\begin{array}{l}\omega \\
5\end{array}\right)$ & & 13 & if $m=39$. \\
\hline
\end{tabular}




\section{ACKNOWLEDGEMENTS}

The authors would like to express their appreciation to all referees for their effort and kind comments for this paper.

\section{REFERENCES}

[1] M. Bhargava, On the Conway-Schneeberger fifteen theorem, Contemp. Math. 272 (2000), 27-37. MR $1803359(2001 \mathrm{~m}: 11050)$

[2] H. Brandt, O. Intrau, Tabellen reduzierter positiver ternärer quadratischer Formen, Akademie-Verlag, Berlin, 1958. MR0106204 (21:4938)

[3] W. K. Chan, M.-H. Kim, S. Raghavan, Ternary universal integral quadratic forms over real quadratic fields, Japan. J. Math. 22 (1996), 263-273. MR.1432376 (97m:11051)

[4] J. H. Conway, Universal quadratic forms and the fifteen theorem, Contemp. Math. 272 (2000), 23-26. MR.1803358 (2001m:11049)

[5] L. E. Dickson, Integers represented by positive ternary quadratic forms, Bull. Amer. Math. Soc. 33 (1927), 63-70. MR.1561323

[6] A. G. Earnest, A. Khosravani, Universal binary Hermitian forms, Math. Comp. 66 (1997), 1161-1168. MR1422787 (98a:11048)

[7] F. Götzky, Uber eine Zahlentheoretische Anwendung von Modulfunktionen zweier Veränderlicher, Math. Ann. 100 (1928), 411-437. MR1512493

[8] J. Hanke, Some recent results about (ternary) quadratic forms, Number theory, CRM Proc. Lecture Notes, 36 (2004), 147-164. MR2076591 (2005k:11076)

[9] J. Hanke, http://www.math.duke.edu/ ${ }^{\sim}$ jonhanke/290/Universal-290.html.

[10] H. Iwabuchi, Universal binary positive definite Hermitian lattices, Rocky Mountain J. Math. 30 (2000), no.3, 951-959. MR.1797825 (2002c:11043)

[11] N. Jacobson, A note on hermitian forms, Bull. Amer. Math. Soc. 46 (1940), 264-268. MR0001957 (1:325d)

[12] B. M. Kim, Positive universal forms over totally real fields, Ph.D. Thesis, Seoul National Univ., 1997.

[13] B. M. Kim, Finiteness of real quadratic fields which admit positive integral diagonal septanary universal forms, Manuscripta Math. 99 (1999), 181-184. MR1697212 (2000h:11032)

[14] B. M. Kim, Universal octonary diagonal forms over some real quadratic fields, Comment. Math. Helv. 75 (2000), 410-414. MR.1793795 (2001m:11046)

[15] B. M. Kim, M.-H. Kim, B.-K. Oh, 2-universal positive definite integral quinary quadratic forms, Contemp. Math. 249 (1999), 51-62. MR1732349 (2001c:11047)

[16] B. M. Kim, M.-H. Kim, B.-K. Oh, A finiteness theorem for representability of quadratic forms by forms, J. Reine Angew. Math. 581 (2005), 23-30. MR2132670 (2006a:11044)

[17] J.-H. Kim, P.-S. Park, A few uncaught universal Hermitian forms, Proceedings of Amer. Math. Soc. 135 (2007), 47-49. MR2280173 (2007i:11055)

[18] M.-H. Kim, Recent developments on universal forms, Contemp. Math. 344 (2004), 215-228. MR2058677 (2005c:11042)

[19] J. L. Lagrange, Démonstration d'un théorème d'arithmétique, CEuvres 3 (1770), 189-201.

[20] H. Maass, Über die Darstellung total positiver Zahlen des Körpers $\mathbf{R}(\sqrt{5})$ als Summe von drei Quadraten, Abh. Math. Sem. Hamburg 14 (1941), 185-191. MR0005505 (3:163a)

[21] O. T. O'Meara, Introduction to Quadratic Forms, Springer-Verlag, Berlin, 1973.

[22] P.-S. Park, 2-Universal Hermitian Forms, Ph.D. Thesis, Seoul National Univ., 2005.

[23] S. Ramanujan, On the expression of a number in the form $a x^{2}+b y^{2}+c z^{2}+d w^{2}$, Proc. Cambridge Phil. Soc. 19 (1917), 11-21.

[24] A. Rokicki, Finiteness results for definite $n$-regular and almost $n$-regular hermitian forms, Ph.D. Thesis, Wesleyan Univ., 2005.

[25] W. A. Schneeberger, Arithmetic and Geometry of Integral Lattices, Ph.D. Thesis, Princeton Univ., 1995.

[26] M. F. Willerding, Determination of all classes of positive quaternary quadratic forms which represent all (positive) integers, Bull. Amer. Math. Soc. 54 (1948), 334-337. MR0024939 $(9: 571 \mathrm{e})$ 
Department of Mathematics, Kangnung-Wonju National University, 123 ChibyonDong, Gangneung, Gangwon-Do, Korea

E-mail address: kbm@nukw.ac.kr

School of Mathematics, Korea Institute for Advanced Study, Hoegiro 87, DongDaemun-Gu, Seoul, 130-722, Korea

E-mail address: jykim@kias.re.kr

Department of Mathematics Education, Kyungnam University, Masan, Kyungnam, 631-701, KOREA

E-mail address: pspark@kyungnam.ac.kr 